\title{
Low fertilizer inputs do not adversely affect yield or performance of Indica hybrid rice
}

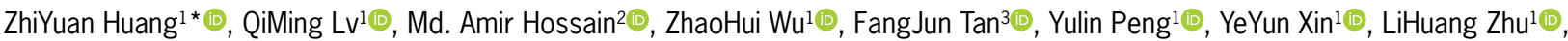 \\ Longping Yuan ${ }^{1+}$ (우
}

\author{
'Hunan Hybrid Rice Research Center, State Key Laboratory \\ of Hybrid Rice, 736 Yuanda Er Road, Furong District, \\ Changsha, Hunan - 410125 - Changsha - China. \\ ${ }^{2}$ Bangladesh Agricultural University - Dept. of Genetics \& \\ Plant Breeding, 2202 - Mymensingh - Bangladesh. \\ 3Hunan Academy of Agricultural Sciences/Negetable \\ Research Institute, 892 Yuanda Er Road - Furong District - \\ 410125 - Changsha - China. \\ †In Memoriam \\ *Corresponding author <huangzhiyuan@hhrrc.ac.cn>
}

Edited by: Francesco Montemurro

Received December 30, 2020

Accepted March 22, 2021
ABSTRACT: The enhancement of rice production numbers can be achieved by using quality rice cultivars and fertilizers. The double rice cropping model has given rise to an important rice production system in southern China. Exploring the possibility of whether hybrid vigor could make a substantial contribution to early and late season rice production, and how the heterosis expression of hybrid rice functions under different levels of fertilizer application is of great significance. The objective of this study was to evaluate the grain yield and associated plant traits of popular hybrid and inbred rice varieties with large-scale promotion under conditions of customary (high) and combined (low) fertilization in the early and late seasons of 2017-18 in Changsha County, Hunan Province, China. We found that hybrid rice varieties displayed their respective advantages in the early and late rice seasons, but the advantages in their relative yield traits varied. The leading advantages of early season rice were effective panicle number per hill (EPN), 1000-grain weight (KGW), harvest index (HI), yield, and nitrogen use efficiency (NUE), whereas in late season rice, the foremost advantages were grain number per panicle (GNP), HI, yield, and NUE. The EPN was the prime advantage of early season hybrid rice with a short growth period, and the GNP was the main advantage of late season hybrid rice with a long growth period. Notably, the main yield advantage of hybrid rice was stronger under combined (low) fertilization than under customary (high) fertilization. Hence, high yield can be achieved by selecting the best hybrid rice varieties supported by combined fertilization (lower fertilizer use with higher efficiency).

Keywords: rice, heterosis, main yield trait, restorer lines, double cropping rice

List of abbreviations: $\mathrm{HCK}=$ Standard heterosis. CUF = Customary fertilization. COF = Combined fertilization. EPN = Effective panicle number. GNP = Grain number per panicle. SSR = Seed set rate. $\mathrm{KGW}=$ The 1000-grain weight. $\mathrm{HI}=$ Harvest index. $\mathrm{PFP}_{\mathrm{N}}=$ Grain yield with $\mathrm{N}$ application / $\mathrm{N}$ application rate

\section{Introduction}

Rice (Oryza sativa L.) is one of the most important food crops, accounting for $25 \%$ of China's total planted area and $37 \%$ of its national cereal production. China will require approximately $20 \%$ more rice production by 2030 to meet its domestic needs if rice consumption per capita maintains its current trends (Cai and Chen, 2000). As such, there are two primary intensification options: (1) increasing rice yield through management optimization and genetic improvement (Neumann et al., 2010); and (2) using existing cropland more frequently each year through multiple cropping (Ray and Foley, 2013). With the development of increases in rice yield, rice varieties have also experienced the transformation from tall stem to dwarf and from inbred rice to hybrid rice (Zou, 2011). It is of great importance to explore whether hybrid vigor could substantially contribute to early and late season rice production and how the heterosis expression of hybrid rice functions under different fertilization conditions.

However, the enthusiasm of farmers to grow double season rice has waned due to high labor input and low economic returns (Peng et al., 2004). Furthermore, excessive use of chemical fertilizer generates a great number of environmental side effects, such as water pollution, eutrophication, high $\mathrm{NO}_{3}^{-}$in underground water, and air pollution (Carpenter 2008; Liu et al., 2013; Norse and Ju, 2015; Zhou et al., 2016) as well as increased greenhouse emissions (Arunrat et al., 2020c) and gray water footprint (Arunrat et al., 2020b). To reduce labor costs in China, several companies have recently developed "medicinal fertilizers". The term "medicinal fertilizer" usually refers to a mixture of pesticides and fertilizers in a given proportion, produced via a special process, which has the integrated function of both the pesticide and the fertilizer. The pesticide can be herbicide, insecticide, or bactericide; the fertilizer can be either a simple or a multi-nutrient compound fertilizer. Farmers can combine the frequently used fertilizer with the medicinal fertilizer to solve the problem of labor shortage. In addition to the maintenance of agricultural growth and increase in yield and harvest, combined fertilization has advantages of both convenience of use and reduced environmental pollution ( $\mathrm{Li}, 2018)$. On the other hand, many farmers select hybrid rice to increase yield, although the performance of early and late season hybrid rice has not been clarified under different fertilization conditions. The objectives of this study were to (1) evaluate the performance of hybrid rice in double cropping seasons, and (2) identify the effects of different fertilization treatments on heterosis expression 
in hybrid versus inbred lines for the purpose of reducing fertilization inputs. We compared several yield related traits, such as biomass accumulation, main yield traits, grain yields, and nitrogen use efficiency (NUE), between tested early and late season hybrid and inbred rice varieties under two fertilization treatments. This work provides valuable data for growers, extension specialists, and fertilizer producers that can be used to maximize yields with reduced fertilizer and pesticide inputs.

\section{Materials and Methods}

\section{Experimental design and operation}

The field experiments were conducted at Lukou rice experimental base $\left(28^{\circ} 12^{\prime} \mathrm{N}, 112^{\circ} 59^{\prime} \mathrm{E}\right.$, altitude of 73 $\mathrm{m})$, Changsha County, Hunan Province, China in 201718 under the condition of subtropical climate (Figure 1). The soil type of the experiment site was red and yellow soil, and the soil characters before the test were total nitrogen $1.64 \mathrm{~g} \mathrm{~kg}^{-1}$, total phosphorus $0.5 \mathrm{~g} \mathrm{~kg}^{-1}$, total potassium $22.71 \mathrm{~g} \mathrm{~kg}^{-1}, \mathrm{pH} 5.90$, organic matter 22.42 $\mathrm{g} \mathrm{kg}^{-1}$, alkali-hydro nitrogen $82.83 \mathrm{mg} \mathrm{kg}^{-1}$ The early season and late season hybrid rice varieties tested in this study were ZLY89, LLY268, TY390 and CLY7, and inbred varieties were ZZ39, XZX45, HHZ and X2 respectively. A detailed description of each variety is given in Table 1. The split plot experimental field covered an area of 0.3 ha. Customary fertilization (CUF: high fertilizer use) and combined fertilization (COF: low fertilizer

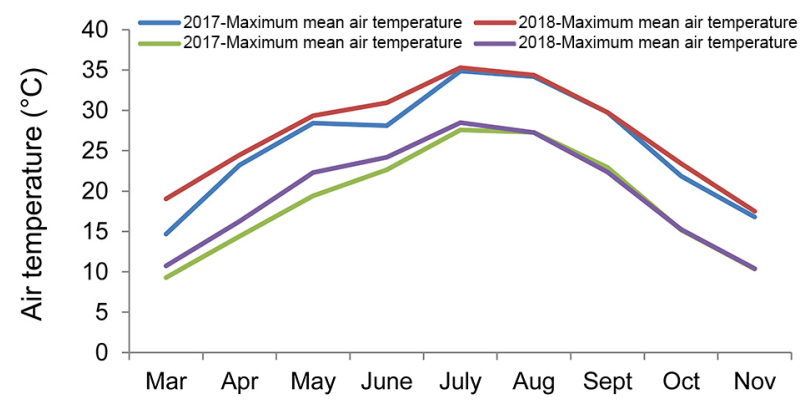

Figure 1 - Average air temperature of the experimental site during rice growing season in 2017 and 2018. Data from the National Meteorological Center of China. use) treatments were applied. Customary fertilization of early and late season rice in 2017 and 2018 included a basal fertilizer application before transplanting, and topdressing approximately one week after transplanting. The detailed fertilizer application amount is listed in Table 2 . The combined fertilization of early and late season rice in 2017 and 2018 included a basal fertilizer application before transplanting, two topdressings with medicinal fertilizer application about one week after transplanting, and during the booting and heading stages, as shown in Table 2. The total fertilizer amount $\left(\mathrm{N}-\mathrm{P}_{2} \mathrm{O}_{5}-\mathrm{K}_{2} \mathrm{O}\right)$ used for customary fertilization of early season rice was 350.84 and $399.82 \mathrm{~kg} \mathrm{ha}^{-1}$ in 2017 and 2018, respectively, and the total fertilizer amount $\left(\mathrm{N}-\mathrm{P}_{2} \mathrm{O}_{5}-\mathrm{K}_{2} \mathrm{O}\right)$ used for combined fertilization of early season rice was 221.67 and 305.34 $\mathrm{kg} \mathrm{ha}^{-1}$ in 2017 and 2018, respectively. The total fertilizer $\left(\mathrm{N}-\mathrm{P}_{2} \mathrm{O}_{5}-\mathrm{K}_{2} \mathrm{O}\right)$ amount of customary fertilization used for late season rice was 443.25 and $399.82 \mathrm{~kg} \mathrm{ha}^{-1}$ in 2017 and 2018, respectively, and the total fertilizer $\left(\mathrm{N}-\mathrm{P}_{2} \mathrm{O}_{5}-\right.$ $\mathrm{K}_{2} \mathrm{O}$ ) amount of combined fertilization used for the late season rice was 221.67 and $291.67 \mathrm{~kg} \mathrm{ha}^{-1}$ in 2017 and 2018, respectively (Table 2). The medicinal fertilizer XSL (N $16 \%$, bensulfuron $0.032 \%$, butachlor $0.608 \%$, China Patent No: ZL201410082830.3) was developed by a local company, which was used as a topdressing fertilizer to control weeds at the rice tillering stage. The medicinal fertilizer SAK (N $20 \%$, furosemide $0.1 \%$ ), also developed by the above company, was to control insects in the paddy field. The date of sowing and transplanting and the specifications for transplanting, number of transplanted seedlings per hill, and maturity of each variety are presented in Table 3. According to the local farmer, field management practice, under customary fertilization conditions, was to spray the paddy rice with pesticides 2-3 times during the entire growth period, although under combined fertilization conditions, the paddy rice was not sprayed with any pesticides. Each treatment area was approximately $30 \mathrm{~m}^{2}$, and the treatments were replicated three times.

\section{Sampling and measurements}

Biomass accumulation was investigated at different stages. After the maturity stage, 20 hills of each variety were sampled for EPN and plant height (PH). Six hills

Table 1 - Rice varieties used for yield comparison tests.

\begin{tabular}{llccc}
\hline Variety name & \multicolumn{1}{c}{ Type } & Male sterile line & Restorer line & Approval status $^{\text {a }}$ \\
\hline ZZ39 & Early season inbred rice & & Main traits & National approved 2012015 \\
XZX45 & Early season inbred rice & & Hunan yield \\
ZLY819 & Early season hybrid rice & Zhu 1S & Hua 819 & Hunan approved 2007002 \\
LLY268 & Early season hybrid rice & Xiangling 628S & Hua 268 & National approved 2008008 \\
HHZ & Late season inbred rice & & High yield & Hunan approved 2007002 \\
X2 & Late season inbred rice & & Hunan approved 2007002 & High yield \\
TY390 & Late season hybrid rice & Taifeng A & Guanghui 390 & Hunan approved 2007002 \\
CLY7 & Late season hybrid rice & C815S & R777 & Hunan approved 2007002 \\
\hline
\end{tabular}

${ }^{a}$ Approval for release by year and by the Crops Variety Approval Committee at or above the provincial level in China. (http://www.ricedata.cn/). 
Table 2 - Different fertilization treatments of early-season and late-season rice.

\begin{tabular}{|c|c|c|c|c|c|c|c|}
\hline $\begin{array}{l}\text { Year-Season- } \\
\text { Fertilization }\end{array}$ & BF: Amount & TF (TS): Amount & TF (BHS): Amount & Total N & $\begin{array}{l}\text { Total } \\
\mathrm{P}_{2} \mathrm{O}_{5}\end{array}$ & $\begin{array}{l}\text { Total } \\
\mathrm{K}_{2} \mathrm{O} f\end{array}$ & $\begin{array}{c}\text { Total } \\
\text { fertilization }\end{array}$ \\
\hline & & $\mathrm{kg} \mathrm{ha}^{-1}$ & & & & & \\
\hline 2017-ESR-CUF & CF (20 \%-8 \%-12 \%): 583.33 & UR (N46 \%) + PF (K $\left.\mathrm{K}_{2} \mathrm{O} 60 \%\right) 125+100$ & & 174.17 & 46.67 & 130 & 350.84 \\
\hline 2017-ESR-COF & $\begin{array}{l}\text { CF }(22-8 \%-12 \%)+\text { RSC: } 416.67 \\
\text { and } 450\end{array}$ & COF1 (16-0.032-0.608): 166.67 & COF2 (20 \%-0.1 \%): 100 & 138.34 & 33.33 & 50 & 221.67 \\
\hline 2018-ESR-CUF & CF (18 \%-4 \%-9 \%): 750 & $\begin{array}{l}\text { UR }(46 \%)+P F\left(K_{2} 060 \%\right): 233.3+ \\
100\end{array}$ & & 242.32 & 30 & 127.5 & 399.82 \\
\hline 2018-ESR-COF & CF $(22-8 \%-12 \%)+$ RSC: $600+450$ & COF1 (16-0.032-0.608): 166.67 & COF2 (20 \%-0.1 \%): 100 & 185.34 & 48 & 72 & 305.34 \\
\hline 2017-LSR-CUF & CF $(15 \%-15 \%-15 \%) 750$ & UR (46 \%) + PF (60 \%): $112.5+90$ & & 164.25 & 112.5 & 166.5 & 443.25 \\
\hline 2017-LSR-COF & $\begin{array}{l}\text { CF }(22-8 \%-12 \%)+\text { RSC: } 416.67+ \\
450\end{array}$ & COF1 (16-0.032-0.608): 166.67 & COF2 (20 \%-0.1 \%): 100 & 138.34 & 33.33 & 50 & 221.67 \\
\hline 2018-LSR-CUF & CF (18 \%-4 \%-9 \%): 750 & UR (46 \%) + PF (60 \%): $233.3+100$ & & 242.32 & 30 & 127.5 & 399.82 \\
\hline 2018-LSR-COF & $\begin{array}{l}\text { CF }(22-8 \%-12 \%)+\text { RSC: } 583.63+ \\
450\end{array}$ & COF1 (16-0.032-0.608): 166.67 & COF2 (20 \%-0.1 \%): 100 & 175.1 & 46.67 & 70 & 291.67 \\
\hline
\end{tabular}

$\overline{\mathrm{ESR}}=$ early season rice; $\mathrm{LSR}=$ late season rice; CUF = customary fertilization; $\mathrm{COF}=$ combined fertilization; $\mathrm{BF}=$ base fertilizer; $\mathrm{TF}=$ topdressing fertilizer; $\mathrm{TS}=$ tillering stage; UR = Urea; $\mathrm{BHS}=$ booting and heading stage; $\mathrm{CF}=$ compound fertilizer $\left(\mathrm{N}: \mathrm{P}_{2} \mathrm{O}_{5}: \mathrm{K}_{2} \mathrm{O}\right)$; COF1 = combined fertilizer 1 ( $\mathrm{N} 16 \%$, bensulfuron $0.032 \%$, butachlor $0.608 \%$ ), COF2 = combined fertilizer 2 (N $20 \%$, furosemide $0.1 \%$ ).

Table 3 - Transplanting specifications for different rice varieties.

\begin{tabular}{lcccccc}
\hline Variety name & Transplanting specifications & Plant number per hill & Sowing date & Transplanting date & Fully-ripened stage date & Growth duration \\
\hline ZZ39 & $\mathrm{cm}$ & & & & & \\
ZZ39 & $16.5 \times 19.8$ & $3-5$ & $25 / 3 / 2017$ & $23 / 4 / 2017$ & $16 / 7 / 2017$ & 113 \\
XZX45 & $16.5 \times 19.8$ & $3-5$ & $25 / 3 / 2018$ & $19 / 4 / 2018$ & $17 / 7 / 2018$ & 114 \\
XZX45 & $16.5 \times 19.8$ & $3-5$ & $25 / 3 / 2017$ & $23 / 4 / 2017$ & $16 / 7 / 2017$ & 113 \\
ZLY819 & $16.5 \times 19.8$ & $3-5$ & $25 / 3 / 2018$ & $19 / 4 / 2018$ & $17 / 7 / 2018$ & 114 \\
ZLY819 & $16.5 \times 19.8$ & $3-5$ & $25 / 3 / 2017$ & $23 / 4 / 2017$ & $16 / 7 / 2017$ & 113 \\
LLY268 & $16.5 \times 19.8$ & $3-5$ & $25 / 3 / 2018$ & $19 / 4 / 2018$ & $17 / 7 / 2018$ & 114 \\
LLY268 & $16.5 \times 19.8$ & $3-5$ & $25 / 3 / 2017$ & $23 / 4 / 2017$ & $16 / 7 / 2017$ & 113 \\
HHZ & $16.5 \times 19.8$ & $3-5$ & $25 / 3 / 2018$ & $19 / 4 / 2018$ & $17 / 7 / 2018$ & 114 \\
HHZ & $19.8 \times 19.8$ & $3-5$ & $18 / 6 / 2017$ & $28 / 7 / 2017$ & $6 / 11 / 2017$ & 141 \\
X2 & $19.8 \times 19.8$ & $3-5$ & $20 / 6 / 2018$ & $19 / 7 / 2018$ & $1 / 11 / 2018$ & 134 \\
X2 & $19.8 \times 19.8$ & $3-5$ & $18 / 6 / 2017$ & $28 / 7 / 2017$ & $10 / 11 / 2017$ & 145 \\
TY390 & $19.8 \times 19.8$ & $3-5$ & $20 / 6 / 2018$ & $19 / 7 / 2018$ & $5 / 11 / 2018$ & 138 \\
TY390 & $19.8 \times 19.8$ & $3-5$ & $18 / 6 / 2017$ & $28 / 7 / 2017$ & $6 / 11 / 2017$ & 141 \\
CLY7 & $19.8 \times 19.8$ & $3-5$ & $20 / 6 / 2018$ & $19 / 7 / 2018$ & $1 / 11 / 2018$ & 134 \\
CLY7 & $19.8 \times 19.8$ & $3-5$ & $18 / 6 / 2017$ & $28 / 7 / 2017$ & $1 / 11 / 2017$ & 136 \\
\hline
\end{tabular}

of each variety were investigated for GNP, seed set rate (SSR), 1000-grain weight (KGW), and harvest index (HI). The panicles of each hill were threshed by hand, and the filled grains separated from unfilled grains by winnowing. The GNP was calculated as the total number of grains divided by the EPN. The SSR was 100 $\times$ total filled grains / number of total grains per hill, and the KGW was $1000 \times$ total filled grains' weight $/$ number of filled grains per hill. Altogether, the above ground biomass was calculated by the sum of dry weight of leaves, stems, rachis, and filled, half-filled, and empty spikelets. The biomass of six hills for each variety was measured after the plants had been heated at $70^{\circ} \mathrm{C}$ for $48 \mathrm{~h}$ to constant weight. The HI (filled grains' weight / total above ground biomass) was calculated separately (Miao et al., 2011). The yield was determined from more than 300 hills in each plot using a combine harvester once the grains had been adjusted to a moisture content of $12 \%$. The HI was calculated as the ratio of the filled grain dry weight to the total above ground biomass. The NUE, defined as the yield produced per unit of $\mathrm{N}$ applied (Cassman et al., 1996; Fageria et al., 2005), was approximate using an $\mathrm{N}$ partial factor productivity $\left(\mathrm{PFP}_{\mathrm{N}}, \mathrm{kg}\right.$ rice grain per $\mathrm{kg} \mathrm{N}$ applied) since it integrates fertilizer input, inherent soil $\mathrm{N}$ supply capacity, and achieved yield, and as such is the broadest measure of NUE (van Noordwijk et al., 2014; Xie et al., 2020). PFP $_{\mathrm{N}}$ $=$ Grain yield with $\mathrm{N}$ application / $\mathrm{N}$ application rate. Standard heterosis (HCK) was calculated according to the following formula:

$H C K=(F 1-C K) / C K \times 100$

where $F 1$ is the performance of yield traits of the hybrid rice, and $C K$, the performance of yield traits of the inbred control variety of early and late season rice. 


\section{Data Analysis}

The data for rice attributes, i.e., biomass accumulation per hill (BPH), PH, EPN, GNP, SSR, KGW, HI, yield, and NUE were subjected to an analysis of variance using the SPSS 17.0 software program. Means of varieties were compared based on the least significant difference test (LSD) at $p \geq 0.05$ probability levels under different fertilization treatments during different seasons and years. The tables and figures were prepared by Microsoft Excel.

\section{Results}

\section{BPH and PH are independent of fertilizer regimen and cultivar across seasons}

Source capacity is typically expressed as a unit of biomass production, which directly relates to the photosynthetic capacity of plants (Zhang et al., 2009). The most common approach for increasing productivity in rice is to increase biomass production (Peng et al., 2009; Yuan, 2012). To evaluate differences in biomass production related to low versus high fertilizer treatments, we analyzed the total BPH (Biomass accumulation per hill) of different cultivars of early and late season rice. It was found that early season rice accumulated more biomass in its late growth stages, whereas late season rice accumulated more biomass after full heading (Tables 4 and 5, Figure 2). Compared with the inbred varieties, the HCK (Standard heterosis) for BPH of early season hybrid rice was largely negative. The difference in $\mathrm{BPH}$ for these hybrids was inconsistent between the two fertilization treatments. Specifically, the HCK for BPH of late season hybrid rice at the booting and maturity stages showed an increase over the inbred cultivars in 2017, but was lower than that of inbred cultivars in 2018 (Tables 6 and 7 , Figure 3). Furthermore, the hybrid rice varieties did not exhibit consistent differences in BPH at different growth stages. Similarly, differences in BPH under fertilization treatments were also non-significant in 2017. However, among late season rice, combined fertilization led to lower biomass accumulation than that of customary fertilization during the full heading and full ripening stages.

Plant height $(\mathrm{PH})$ was relatively lower among early season rice than late season rice, averaging $70-80 \mathrm{~cm}$. The $\mathrm{PH}$ of early season rice was 83.06 and $84.89 \mathrm{~cm}$ in 2017 and 78.43 and $79.76 \mathrm{~cm}$ in 2018 under CUF and COF treatments, respectively (Tables 8 and 9, Figure 4). The average HCK for $\mathrm{PH}$ of early season hybrid rice varieties was $0.05 \%$ and $1.65 \%$ in 2017, and $3.12 \%$ and $2.63 \%$ in 2018 under the CUF and COF treatments, respectively, indicating a better performance of the hybrid rice. Under the COF treatment, the average $\mathrm{PH}$ of early season hybrid rice varieties was higher than that under the CUF treatment (Tables 10 and 11, Figure 5).

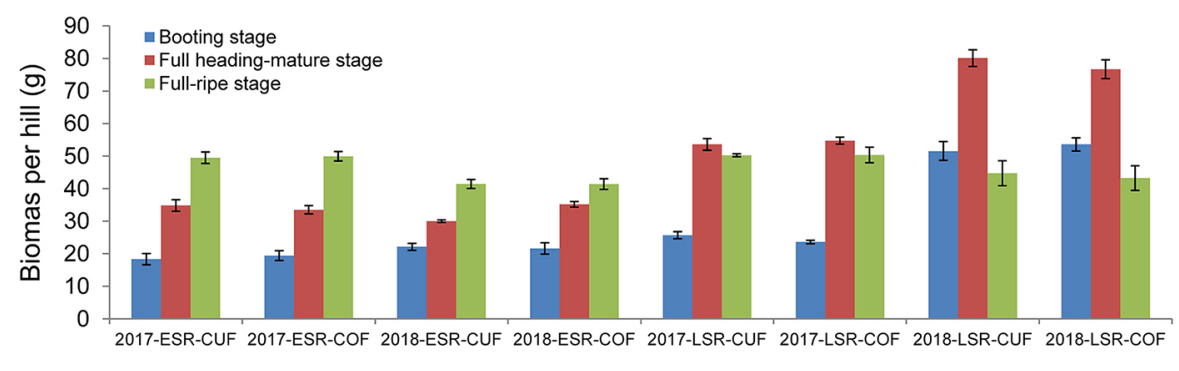

Fertilizattion treatments during different seaons

Figure 2 - Biomass accumulation of early season and late season rice under different fertilization conditions. ESR = early season rice; LSR = late season rice; CUF = customary fertilization; $\mathrm{COF}=$ combined fertilization.

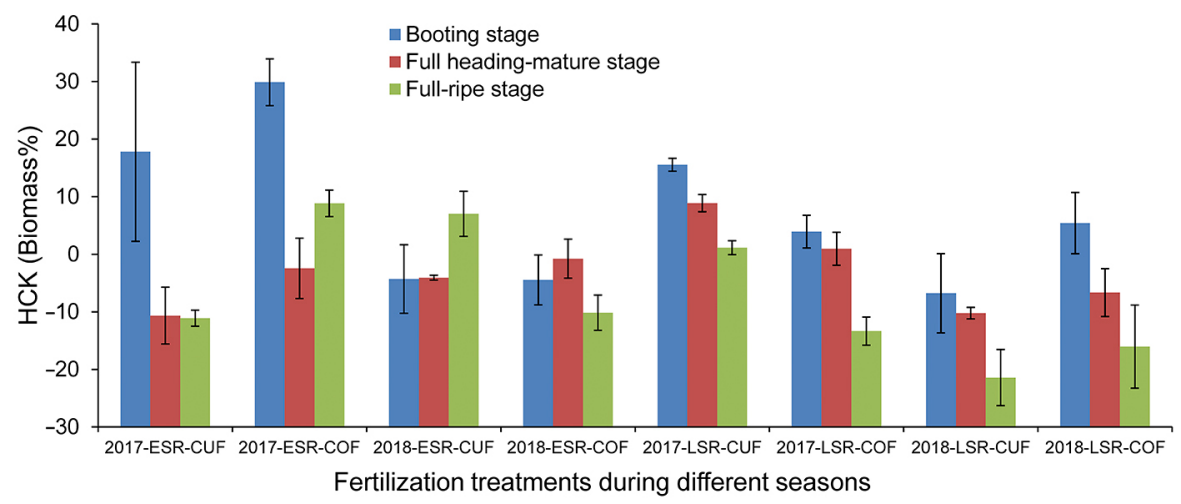

Figure 3 - The standard heterosis (HCK) for biomass accumulation of early seasonand late season hybrid rice under different fertilization conditions. ESR = early season rice; LSR = late season rice; CUF = customary fertilization; COF = combined fertilization. 
Table 4 - Biomass accumulation of early-season rice and late-season rice in different stages.

\begin{tabular}{|c|c|c|c|}
\hline \multirow[b]{2}{*}{ Year-season-variety-fertilization } & Booting stage (8/6/2017) & Yellow mature stage $(10 / 7 / 2017)$ & Fully-ripened stage $(15 / 7 / 2017)$ \\
\hline & $\begin{array}{l}\text { Biomass per hill (g) } \\
(\text { Mean } \pm \text { SE) }\end{array}$ & $\begin{array}{c}\text { Biomass per hill (g) } \\
(\text { Mean } \pm \text { SE) }\end{array}$ & $\begin{array}{c}\text { Biomass per hill }(\mathrm{g}) \\
(\text { Mean } \pm \text { SE) }\end{array}$ \\
\hline 2017-ESR-ZZ39-CUF & $13.37 \pm 1.72 d$ & $34.5 \pm 2.40 a b$ & $52.9 \pm 4.79 a$ \\
\hline 2017-ESR-ZZ39-COF & $16.25 \pm 1.61 \mathrm{~cd}$ & $32.99 \pm 2.31 \mathrm{ab}$ & $48.01 \pm 0.73 \mathrm{a}$ \\
\hline 2017-ESR-XZX45-CUF & $21.3 \pm 1.61 \mathrm{ab}$ & $39.22 \pm 3.55 a$ & $51.96 \pm 2.03 a$ \\
\hline 2017-ESR-XZX45-COF & $17.58 \pm 1.54 \mathrm{bcd}$ & $34.92 \pm 3.33 a b$ & $47.69 \pm 4.96 a$ \\
\hline 2017-ESR-ZLY819-CUF & $19.46 \pm 1.40 \mathrm{abc}$ & $30.48 \pm 1.69 b$ & $47.8 \pm 3.9 \mathrm{a}$ \\
\hline 2017-ESR-ZLY819-COF & $22.75 \pm 2.37 \mathrm{a}$ & $30.16 \pm 1.42 b$ & $53.97 \pm 5.04 a$ \\
\hline 2017-ESR-LLY268-CUF & $19.24 \pm 1.71 \mathrm{abc}$ & $35.12 \pm 4.72 a b$ & $45.41 \pm 4.86 a$ \\
\hline 2017-ESR-LLY268-COF & $21.11 \pm 1.92 \mathrm{abc}$ & $36.02 \pm 0.82 a b$ & $50.18 \pm 2.84 a$ \\
\hline \multirow[t]{3}{*}{ Mean } & 18.88 & 34.18 & 49.74 \\
\hline & Booting stage (7/6/2018) & Yellow mature stage $(27 / 6 / 2018)$ & Fully-ripened stage $(12 / 7 / 2018)$ \\
\hline & $\begin{array}{l}\text { Biomass per hill }(\mathrm{g}) \\
\text { (Mean } \pm \mathrm{SE} \text { ) }\end{array}$ & $\begin{array}{l}\text { Biomass per hill }(\mathrm{g}) \\
(\text { Mean } \pm \mathrm{SE})\end{array}$ & $\begin{array}{l}\text { Biomass per hill }(\mathrm{g}) \\
(\text { Mean } \pm \text { SE) }\end{array}$ \\
\hline 2018-ESR-ZZ39-CUF & $20.32 \pm 1.48 b$ & $30.84 \pm 2.83 a b$ & $41.27 \pm 3.27 a$ \\
\hline 2018-ESR-ZZ39-COF & $22.64 \pm 1.84 a b$ & $34.76 \pm 2.7 \mathrm{ab}$ & $45.41 \pm 1.81 \mathrm{a}$ \\
\hline 2018-ESR-XZX45-CUF & $25.15 \pm 2.01 \mathrm{a}$ & $30.44 \pm 1.44 a b$ & $38.88 \pm 3.61 \mathrm{a}$ \\
\hline 2018-ESR-XZX45-COF & $21.64 \pm 1.87 b$ & $35.87 \pm 2.82 a b$ & $41.92 \pm 3.14 \mathrm{a}$ \\
\hline 2018-ESR-ZLY819-CUF & $21.16 \pm 0.89 a b$ & $29.26 \pm 1.67 b$ & $40.45 \pm 2.27 a$ \\
\hline 2018-ESR-ZLY819-COF & $22.73 \pm 0.73 a b$ & $37.04 \pm 1.76 \mathrm{a}$ & $37.47 \pm 3.83 a$ \\
\hline 2018-ESR-LLY268-CUF & $21.86 \pm 1.89 a b$ & $29.51 \pm 2.59 b$ & $45.25 \pm 2.15 a$ \\
\hline 2018-ESR-LLY268-COF & $19.55 \pm 1.42 b$ & $33.03 \pm 2.29 a b$ & $40.85 \pm 2.10 a$ \\
\hline \multirow[t]{3}{*}{ Mean } & 21.88 & 32.59 & 41.44 \\
\hline & Booting stage (3/8/2017) & Yellow mature stage $(10 / 10 / 2017)$ & Fully-ripened stage (30/10/2017) \\
\hline & $\begin{array}{l}\text { Biomass per hill (g) } \\
\text { (Mean } \pm \text { SE) }\end{array}$ & $\begin{array}{l}\text { Biomass per hill (g) } \\
\text { (Mean } \pm \mathrm{SE})\end{array}$ & $\begin{array}{l}\text { Biomass per hill }(\mathrm{g}) \\
(\text { Mean } \pm \mathrm{SE})\end{array}$ \\
\hline 2017-LSR-HHZ-CUF & $24.2 \pm 1.24 \mathrm{a}$ & $48.6 \pm 2.51 \mathrm{a}$ & $51.03 \pm 2.9 a$ \\
\hline 2017-LSR-HHZ-COF & $23.99 \pm 1.65 a$ & $57.48 \pm 7.45 a$ & $56.6 \pm 8.31 \mathrm{a}$ \\
\hline 2017-LSR-X2-CUF & $23.49 \pm 1.8 \mathrm{a}$ & $56.87 \pm 4.75 a$ & $48.98 \pm 2.05 a$ \\
\hline 2017-LSR-X2-COF & $22.33 \pm 1.53 \mathrm{a}$ & $52.53 \pm 3.27 a$ & $51.42 \pm 2.54 \mathrm{a}$ \\
\hline 2017-LSR-TY390-CUF & $27.33 \pm 2.17 a$ & $53.75 \pm 1.62 \mathrm{a}$ & $50.68 \pm 1.6 a$ \\
\hline 2017-LSR-TY390-COF & $23.29 \pm 0.97 a$ & $55.1 \pm 3.82 \mathrm{a}$ & $47.17 \pm 2.51 \mathrm{a}$ \\
\hline 2017-LSR-CLY7-CUF & $27.76 \pm 3.17 \mathrm{a}$ & $55.09 \pm 3.74 a$ & $50.41 \pm 3.58 a$ \\
\hline 2017-LSR-CLY7-COF & $24.79 \pm 1.944 \mathrm{a}$ & $53.77 \pm 7.01 \mathrm{a}$ & $46.21 \pm 3 a$ \\
\hline \multirow[t]{3}{*}{ Mean } & 24.65 & 54.15 & 50.31 \\
\hline & Booting stage $(31 / 8 / 2018)$ & Full heading stage (18/9/2018) & Fully-ripened stage (31/10/2018) \\
\hline & $\begin{array}{l}\text { Biomass per hill (g) } \\
(\text { Mean } \pm \text { SE) }\end{array}$ & $\begin{array}{c}\text { Biomass per hill }(\mathrm{g}) \\
(\text { Mean } \pm \mathrm{SE})\end{array}$ & $\begin{array}{c}\text { Biomass per hill }(\mathrm{g}) \\
(\text { Mean } \pm \mathrm{SE})\end{array}$ \\
\hline 2018-LSR-HHZ-CUF & $49.97 \pm 4.25 \mathrm{ab}$ & $83.32 \pm 4.23 a$ & $45.62 \pm 3.31 b$ \\
\hline 2018-LSR-HHZ-COF & $48.1 \pm 4.96 \mathrm{ab}$ & $75.36 \pm 6.08 b$ & $40.51 \pm 0.88 b c$ \\
\hline 2018-LSR-X2-CUF & $56.99 \pm 2.44 a$ & $85.51 \pm 1.39 \mathrm{a}$ & $55 \pm 2.08 \mathrm{a}$ \\
\hline 2018-LSR-X2-COF & $56.6 \pm 4.88 a$ & $83.53 \pm 3.72 \mathrm{a}$ & $54.5 \pm 2.25 a$ \\
\hline 2018-LSR-TY390-CUF & $55.07 \pm 3.03 a b$ & $74.68 \pm 4.63 a b$ & $37.11 \pm 3.30 c$ \\
\hline 2018-LSR-TY390-COF & $53.03 \pm 3.41 \mathrm{ab}$ & $78.16 \pm 5.93 a b$ & $38.17 \pm 1.69 c$ \\
\hline 2018-LSR-CLY7-CUF & $44.2 \pm 3.14 b$ & $76.85 \pm 5.81 \mathrm{ab}$ & $41.27 \pm 2.15 b c$ \\
\hline 2018-LSR-CLY7-COF & $56.6 \pm 4.88 a$ & $69.74 \pm 3.78 b$ & $39.86 \pm 1.71 b c$ \\
\hline Mean & 52.57 & 78.40 & 44.0 \\
\hline
\end{tabular}

ESR = early season rice; $\mathrm{LSR}=$ late season rice; CUF $=$ customary fertilization; $\mathrm{COF}=$ combined fertilization. Within a column, the same lowercase letters for biomass per hilll among each variety under different fertilization treatments in each season and year, indicate non-significant differences according to the least significant difference test (LSD, $p \geq 0.05$ ).

In contrast, the $\mathrm{PH}$ varied widely among late season rice varieties. The $\mathrm{PH}$ of inbred rice variety $\mathrm{X} 2$ was the highest, reaching $110 \mathrm{~cm}$, whereas that of other varieties averaged $90-100 \mathrm{~cm}$ (Table 8$)$. The $\mathrm{PH}$ of late season rice was 101.79 and $100.86 \mathrm{~cm}$ in 2017 and 103.49 and 105.80 $\mathrm{cm}$ in 2018 under CUF and COF treatments, respectively. Thus, PH was $0.93 \mathrm{~cm}$ and $2.31 \mathrm{~cm}$ higher under CUF treatments in 2017 and 2018, respectively (Tables 8 and 9, Figure 4). Compared with late season inbred rice, the HCK for $\mathrm{PH}$ of late season hybrid rice was $-3.07 \%$ and $-1.55 \%$ in 2017 and $-6.58 \%$ and $-4.94 \%$ in 2018 under CUF and COF treatments, respectively, indicating a decrease in heterosis (Tables 10 and 11, Figure 5). Under the $\mathrm{COF}$ treatment, the average $\mathrm{PH}$ among late season 
Table 5 - The average biomass accumulation between different fertilization conditions.

\begin{tabular}{|c|c|c|c|}
\hline & Booting stage $(8 / 6 / 2017)$ & Yellow maturity $(11 / 7 / 2017)$ & Fully-ripened stage (15/7/2017) \\
\hline Year-season-fertilization & $\begin{array}{l}\text { Biomass per hill (g) } \\
(\text { Mean } \pm \text { SE) }\end{array}$ & $\begin{array}{c}\text { Biomass per hill }(\mathrm{g}) \\
(\text { Mean } \pm \mathrm{SE})\end{array}$ & $\begin{array}{c}\text { Biomass per hill }(\mathrm{g}) \\
(\text { Mean } \pm \mathrm{SE})\end{array}$ \\
\hline 2017-ESR-CUF & $18.34 \pm 1.72$ & $34.83 \pm 1.79$ & $49.52 \pm 1.76$ \\
\hline \multirow[t]{3}{*}{ 2017-ESR-COF } & $19.42 \pm 1.51$ & $33.52 \pm 1.28$ & $49.96 \pm 1.45$ \\
\hline & Booting stage $(7 / 6 / 2018)$ & Yellow maturity $(2 / 6 / 2018)$ & Fully-ripened stage $(1 / 7 / 2018)$ \\
\hline & $\begin{array}{c}\text { Biomass per hill (g) } \\
(\text { Mean } \pm \text { SE) }\end{array}$ & $\begin{array}{c}\text { Biomass per hill (g) } \\
(\text { Mean } \pm \text { SE) }\end{array}$ & $\begin{array}{c}\text { Biomass per hill }(\mathrm{g}) \\
(\text { Mean } \pm \text { SE) }\end{array}$ \\
\hline 2018-ESR-CUF & $22.12 \pm 1.06$ & $30.01 \pm 0.38 \mathrm{a}$ & $41.46 \pm 1.36$ \\
\hline \multirow[t]{3}{*}{ 2018-ESR-COF } & $21.64 \pm 0.74$ & $35.18 \pm 0.85 b$ & $41.41 \pm 1.64$ \\
\hline & Booting stage $(30 / 8 / 2017)$ & Yellow maturity $(10 / 10 / 2017)$ & Fully-ripened stage $(30 / 10 / 2017)$ \\
\hline & $\begin{array}{c}\text { Biomass per hil l(g) } \\
(\text { Mean } \pm \mathrm{SE})\end{array}$ & $\begin{array}{c}\text { Biomass per hill (g) } \\
(\text { Mean } \pm \mathrm{SE})\end{array}$ & $\begin{array}{c}\text { Biomass per hill }(g) \\
(\text { Mean } \pm \text { SE) }\end{array}$ \\
\hline 2017-LSR-CUF & $25.7 \pm 1.08$ & $53.58 \pm 1.78$ & $50.28 \pm 0.45$ \\
\hline \multirow[t]{3}{*}{ 2017-LSR-COF } & $23.6 \pm 0.52$ & $54.72 \pm 1.06$ & $50.35 \pm 2.37$ \\
\hline & Booting stage $(3 / 8 / 2018)$ & Full Heading stage (18/9/2018) & Fully-ripened stage (31/10/2018) \\
\hline & $\begin{array}{l}\text { Biomass per hill (g) } \\
(\text { Mean } \pm \text { SE) }\end{array}$ & $\begin{array}{c}\text { Biomass per hill }(\mathrm{g}) \\
(\text { Mean } \pm \text { SE) }\end{array}$ & $\begin{array}{c}\text { Biomass per hill }(\mathrm{g}) \\
(\text { Mean } \pm \mathrm{SE})\end{array}$ \\
\hline 2018-LSR-CUF & $51.56 \pm 2.87$ & $80.09 \pm 2.58$ & $44.75 \pm 3.83$ \\
\hline \multirow[t]{2}{*}{ 2018-LSR-COF } & $53.58 \pm 2.01$ & $76.7 \pm 2.87$ & $43.26 \pm 3.78$ \\
\hline & $($ Mean $\pm \mathrm{SE})$ & $($ Mean \pm SE) & $($ Mean $\pm \mathrm{SE})$ \\
\hline
\end{tabular}

$\mathrm{ESR}=$ early season rice; $\mathrm{LSR}=$ late season rice; CUF = customary fertilization; $\mathrm{COF}=$ combined fertilization. Within a column, the same lowercase letters for biomass among each variety under different fertilization conditions in each season and year, indicate non-significant differences according to the least significant difference test (LSD, $p \geq 0.05)$.

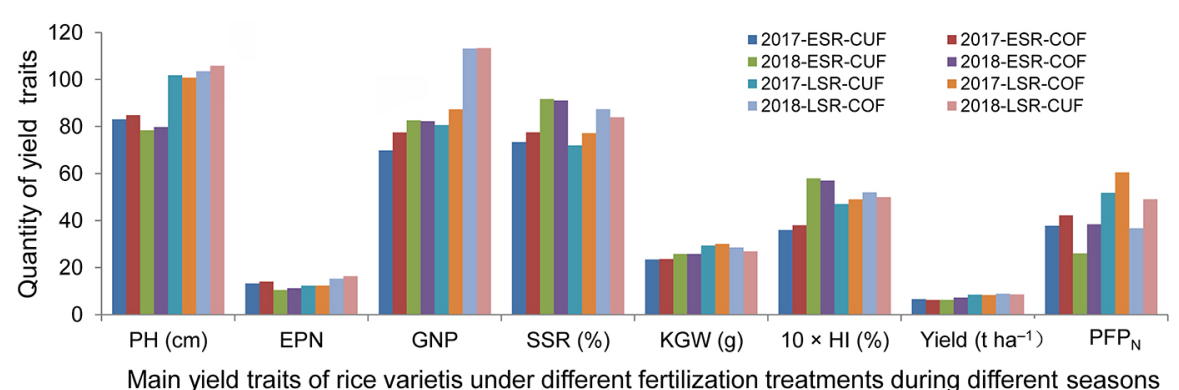

Figure 4 - Performance of traits of different early season and late season rice varieties under different fertilization conditions. ESR = early season rice; $\mathrm{LSR}=$ late season rice; CUF = customary fertilization; $\mathrm{COF}=$ combined fertilization.

hybrid rice varieties was higher on average than that under the CUF treatment, suggesting that higher fertilizer application did not significantly affect plant height as a function of biomass production.

\section{Low fertilization increased EPN per hill in early season hybrids and GNP in late season hybrids}

The Tiller number is also an informative and quantitative agronomic trait in rice as it is positively correlated with panicle number per $\mathrm{m}^{2}$ (Ao et al., 2010; Miller et al., 1991; Wu et al., 1998). EPN represents the tillering ability of varieties and contributes to other yield related traits. The average EPN of early season rice varieties was 13.22 and 13.99 in 2017, and 10.50 and 11.21 in 2018 under CUF and COF treatments, respectively (Tabls 8 and 9, Figure 4). Compared with early season inbred varieties, the average HCK for EPN of early season hybrid rice was $13.27 \%$ and $14.1 \%$ in 2017 and $9.07 \%$ and $7.45 \%$ in 2018 under CUF and COF treatments, respectively (Tables 10 and 11, Figure 5). Taken together, these data indicate that the early season hybrids produced higher EPN than that of inbred lines; however, the higher fertilizer application did not affect EPN.

The average EPN of late season rice varieties was 12.31 and 12.4 in 2017 and 15.32, and 16.34 in 2018 under CUF and COF treatments, respectively (Tables 8 and 9, Figure 4). Compared with late season inbred lines, the average HCK for EPN of late season hybrid rice varieties was $1.86 \%$ and $0.08 \%$ in 2017 and $2.62 \%$ and $-0.29 \%$ in 2018 under CUF and COF treatments, respectively (Tables 10 and 11, Figure 5). These results showed that the EPN of early season hybrids were higher than that of inbred lines, but lower in late season hybrids; furthermore, CUF generally led to greater EPN, although not consistently (e.g., COF improved EPN in 2017 early season rice). 
Table 6 - Standard heterosis (HCK) forbiomass accumulation of early and late season hybrid rice in different stages.

\begin{tabular}{|c|c|c|c|}
\hline \multirow{2}{*}{ Hybrid rice-inbred-year-fertilization } & Booting stage $(8 / 6 / 2017)$ & Yellow mature stage $(11 / 7 / 2017)$ & Fully-ripened stage $(15 / 7 / 2017)$ \\
\hline & HCK (biomass per hill \%) & HCK (biomass per hill \%) & HCK (biomass per hill \%) \\
\hline ZLY819-ZZ39-2017-CUF & $45.55^{*}$ & -11.65 & -9.64 \\
\hline ZLY819-ZZ39-2017-COF & $40^{*}$ & -8.58 & 12.41 \\
\hline ZLY819-XZX45-2017-CUF & -8.64 & $-22.28^{*}$ & -8.01 \\
\hline ZLY819-XZX45-2017-COF & $29.41^{*}$ & -13.63 & 13.17 \\
\hline LLY268-ZZ39-2017-CUF & $43.9^{*}$ & 1.8 & -14.16 \\
\hline LLY268-ZZ39-2017-COF & 29.91 & 9.18 & 4.52 \\
\hline LLY268-XZX45-2017-CUF & -9.67 & -10.45 & -12.61 \\
\hline LLY268-XZX45-2017-COF & 20.08 & 3.15 & 5.22 \\
\hline \multirow[t]{3}{*}{ Mean } & 7.92 & -6.56 & -1.14 \\
\hline & Booting stage (7/6/2018) & Yellow mature stage $(27 / 6 / 2018)$ & Fully-ripened stage $(12 / 7 / 2018)$ \\
\hline & HCK (biomass per hill \%) & HCK (biomass per hill \%) & HCK (biomass per hill \%) \\
\hline ZLY819-ZZ39-2018-CUF & 4.13 & -5.12 & -1.99 \\
\hline ZLY819-ZZ39-2018-COF & 0.4 & 6.56 & -17.49 \\
\hline ZLY819-XZX45-2018-CUF & -15.86 & -3.88 & 4.04 \\
\hline ZLY819-XZX45-2018-COF & 5.04 & 3.26 & -10.62 \\
\hline LLY268-ZZ39-2018-CUF & 7.58 & -4.31 & 9.64 \\
\hline LLY268-ZZ39-2018-COF & -13.65 & -4.98 & -10.04 \\
\hline LLY268-XZX45-2018-CUF & -13.08 & -3.06 & 16.38 \\
\hline LLY268-XZX45-2018-COF & -9.66 & -7.92 & -2.55 \\
\hline \multirow[t]{3}{*}{ Mean } & -4.39 & -2.43 & -1.58 \\
\hline & Booting stage $(30 / 8 / 2017)$ & Yellow mature stage $(10 / 10 / 2017)$ & Fully-ripened stage (30/10/2017) \\
\hline & HCK (biomass per hill \%) & HCK (biomass per hill \%) & HCK (biomass per hill \%) \\
\hline TY390-HHZ-2017-CUF & 12.93 & 5.33 & -0.69 \\
\hline TY390-HHZ-2017-COF & -2.92 & -2.81 & -16.66 \\
\hline TY390-X2-2017-CUF & 16.35 & 9.74 & 3.47 \\
\hline TY390-X2-2017-COF & 4.3 & 6.98 & -8.27 \\
\hline CLY7-HHZ-2017-CUF & 14.71 & 7.96 & -1.21 \\
\hline LLY268-HHZ-2017-COF & 3.33 & -5 & -18.36 \\
\hline LLY268-X2-2017-CUF & 18.18 & 12.47 & 2.92 \\
\hline LLY268-X2-2017-COF & 11.02 & 4.57 & -10.13 \\
\hline \multirow[t]{3}{*}{ Mean } & 9.74 & 4.91 & -6.12 \\
\hline & Booting stage $(31 / 8 / 2018)$ & Full Heading stage (18/9/2018) & Fully-ripened stage (31/10/2018) \\
\hline & HCK (biomass per hill \%) & HCK (biomass per hill \%) & HCK (biomass per hill \%) \\
\hline TY390-HHZ-2018-CUF & 10.21 & -10.37 & $-18.65^{*}$ \\
\hline TY390-HHZ-2018-COF & 10.25 & 3.72 & -5.78 \\
\hline TY390-X2-2018-CUF & -3.37 & -12.67 & $-32.53^{*}$ \\
\hline TY390-X2-2018-COF & -6.31 & -6.43 & $-29.96^{*}$ \\
\hline CLY7-HHZ-2018-CUF & -11.55 & -7.77 & -9.54 \\
\hline LLY268-HHZ-2018-COF & 17.67 & -7.46 & -1.6 \\
\hline LLY268-X2-2018-CUF & $-22.44^{*}$ & -10.13 & $-24.96^{*}$ \\
\hline LLY268-X2-2018-COF & 0 & $-16.51^{\star}$ & $-26.86^{\star}$ \\
\hline Mean & 2.41 & -7.3 & 5.64 \\
\hline
\end{tabular}

ESR CUF = customary fertilization; COF $=$ combined fertilization. Within a column in each season and year, ${ }^{*}$ represents a significant difference between the hybrid variety and inbrids variety, according to the least significant difference test (LSD, $p \geq 0.05$ ).

Several field studies have reported that higher grain yields among hybrid rice varieties are associated with a large sink size (spikelets per $\mathrm{m}^{2}$ ) because of large panicles (GNP) (Huang et al., 2011; Zhang et al., 2009). The average GNP of early rice was 69.87 and 77.48 in 2017 and 82.67 and 82.2 in 2018 under CUF and COF treatments, respectively (Tables 8 and 9, Figure 4). Compared with early season inbred varieties, the average HCK for GNP in the early season hybrid was $-19.89 \%$ and $-5.28 \%$ in 2017 and $-7.71 \%$ and $-6.58 \%$ in 2018 under CUF and COF treatments, respectively (Tables 10 and 11, Figure
4). Given the primarily negative trends in GNP for hybrid varieties, these results indicated that the early season hybrids did not exhibit strong heterosis in GNP and were not affected by lower fertilizer application.

The average GNP of late season rice was 80.58 and 87.32 in 2017 and 113.18 and 113.34 in 2018 under CUF and COF treatments, respectively (Tables 8 and 9, Figure 4). Compared with late season inbred rice varieties, the average HCK for GNP of the late-season hybrids was $16.83 \%$ and $11.68 \%$ in 2017 and $2.31 \%$ and $6.28 \%$ in 2018, under CUF and COF treatments, respectively, 


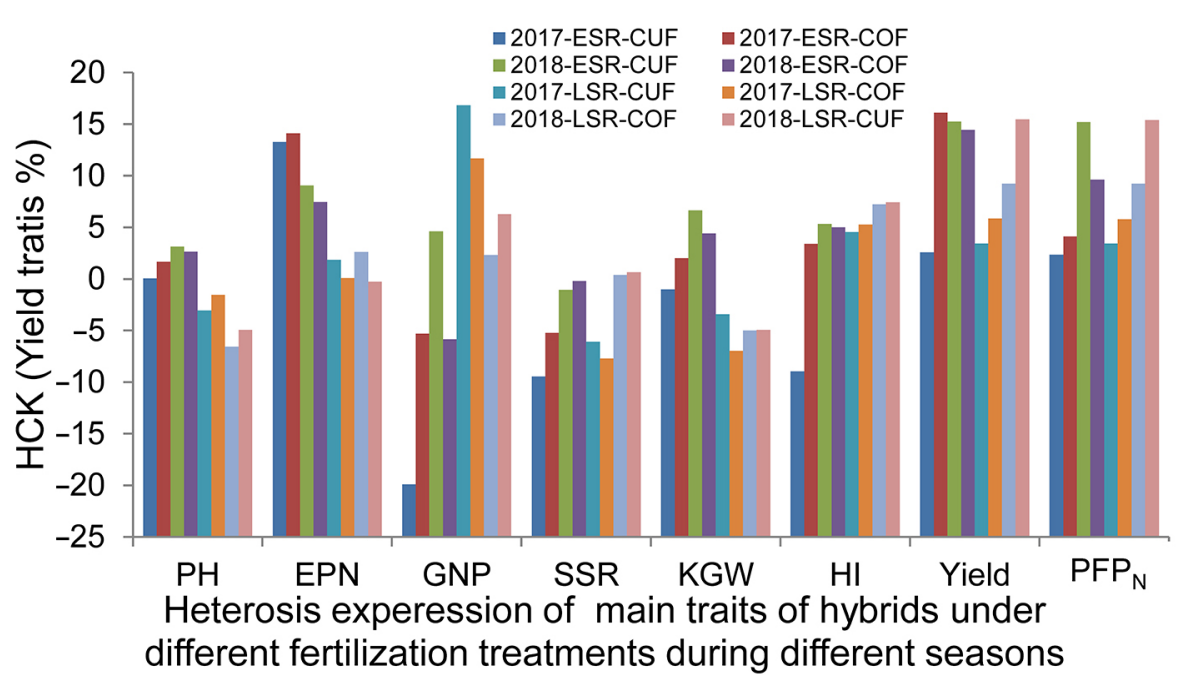

Figure 5 - The standard heterosis $(\mathrm{HCK})$ for main traits of early season and late season rice under different fertilization conditions. $\mathrm{PH}=\mathrm{plant}$ height; EPN = effective panicle number per hill; GNP = grain number per panicle; SSR = seed set rate; KGW = 1000-grain weight; $\mathrm{HI}=$ harvest index; $\mathrm{PFP}_{\mathrm{N}}=\mathrm{N}$ partial factor productivity; $\mathrm{ESR}=$ early season rice; $\mathrm{LSR}=$ late season rice; CUF = customary fertilization; COF = combined fertilization.

Table 7 - Standard heterosis (HCK) for biomass accumulation in early season and late season rice under different fertilization conditions.

\begin{tabular}{|c|c|c|c|}
\hline \multirow{2}{*}{ Year-season-fertilization } & Booting stage (8/6/2017) & Yellow mature stage $(11 / 7 / 2017)$ & Fully-ripened stage (15/7/2017) \\
\hline & HCK (biomass per hill \%) & HCK (biomass per hill \%) & HCK (biomass per hill \%) \\
\hline 2017-ESR-CUF & $17.79 \pm 15.56$ & $-10.65 \pm 4.93$ & $-11.1 \pm 1.39$ \\
\hline \multirow[t]{3}{*}{ 2017-ESR-COF } & $29.85 \pm 4.07$ & $-2.47 \pm 5.24$ & $8.83 \pm 2.30$ \\
\hline & Booting stage $(7 / 6 / 2018)$ & Yellow mature stage $(27 / 6 / 2018)$ & Fully-ripened stage (12/7/2018) \\
\hline & HCK (biomass per hill \%) & HCK (biomass per hill \%) & HCK (biomass per hill \%) \\
\hline 2018-ESR-CUF & $-4.31 \pm 5.94$ & $-4.09 \pm 0.43$ & $7.02 \pm 3.92 b$ \\
\hline \multirow[t]{3}{*}{ 2018-ESR-COF } & $-4.47 \pm 4.33$ & $-0.77 \pm 3.4$ & $-10.17 \pm 3.05 \mathrm{a}$ \\
\hline & Booting stage $(30 / 8 / 2017)$ & Yellow mature stage $(10 / 10 / 2017)$ & Fully-ripened stage (30/10/2017) \\
\hline & HCK (biomass per hill \%) & HCK (biomass per hill \%) & HCK (biomass per hill \%) \\
\hline 2017-LSR-CUF & $15.54 \pm 1.12 b$ & $8.87 \pm 1.50$ & $1.12 \pm 1.21$ \\
\hline \multirow[t]{3}{*}{ 2017-LSR-COF } & $3.93 \pm 2.85 \mathrm{a}$ & $0.94 \pm 2.87$ & $-13.35 \pm 2.45$ \\
\hline & Booting stage $(31 / 8 / 2018)$ & Full Heading stage (18/9/2018) & Fully-ripened stage (31/10/2018) \\
\hline & HCK (biomass per hill \%) & HCK (biomass per hill \%) & HCK (biomass per hill \%) \\
\hline 2018-LSR-CUF & $-6.79 \pm 6.88$ & $-10.23 \pm 1.0$ & $-21.42 \pm 4.87$ \\
\hline 2018-LSR-COF & $5.4 \pm 5.33$ & $-6.67 \pm 4.14$ & $-16.05 \pm 7.21$ \\
\hline
\end{tabular}

indicating positive heterosis (Tables 10 and 11, Figure 5). Thus, the HCK for GNP in late season hybrid varieties was higher under CUF application than under COF, suggesting that this trait was positively affected by higher fertilizer application in the late season hybrids.

\section{Fertilizer regimen significantly affected neither SSR nor KGW for hybrid lines}

Seed set rate (SSR) is also one of the main quantitative traits for assessing yield. We examined differences in SSR to determine the effects of reduced fertilizer application on yield. It was found that the SSR of early season rice was $73.5 \%$ and $77.57 \%$ in 2017 and 91.64 $\%$ and $91.02 \%$ in 2018 under CUF and COF treatments, respectively (Tables 8 and 9, Figure 4). The SSR of early season rice varieties was higher than $90 \%$ in 2018, with negligible differences between varieties but significant differences between years. Compared with early season inbred varieties, the HCK for SSR of early season hybrid rice varieties was $-9.44 \%$ and $-5.23 \%$ in 2017 and $-1.07 \%$ and $-0.2 \%$ in 2018 under conditions of CUF and COF treatments, respectively (Tables 10 and 11, Figure 5). Although all hybrids showed negative heterosis, the 


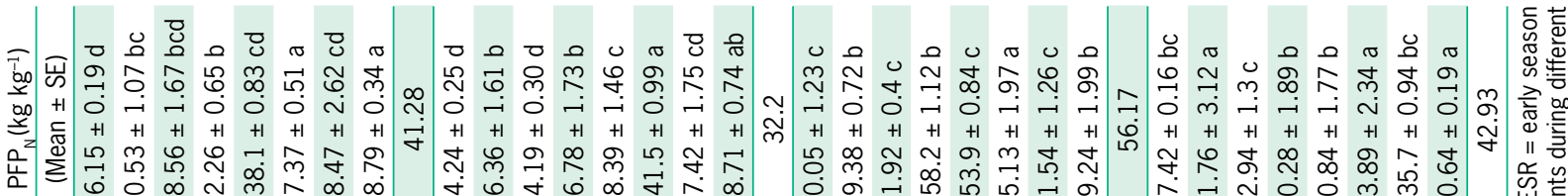

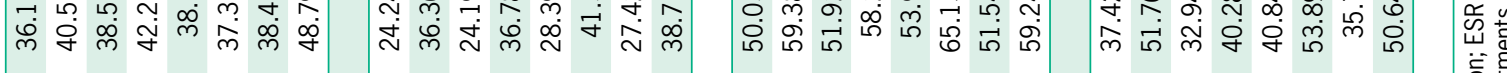

章

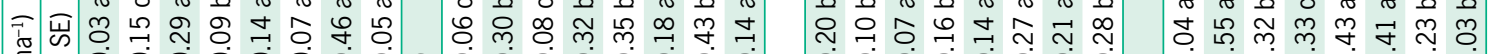

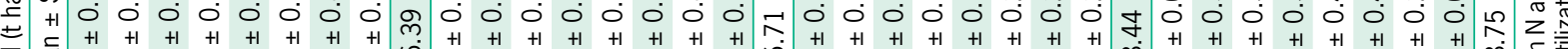

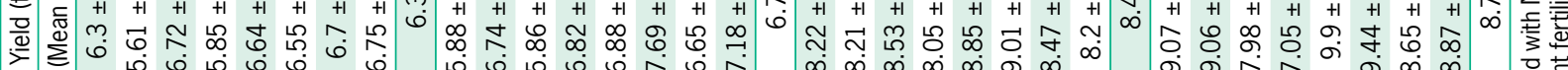

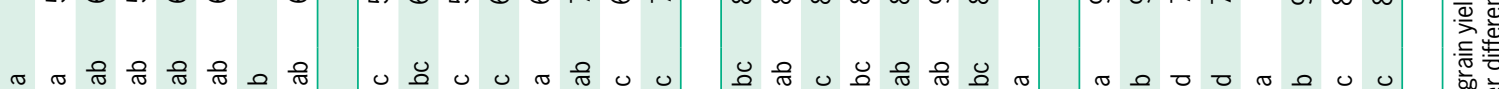

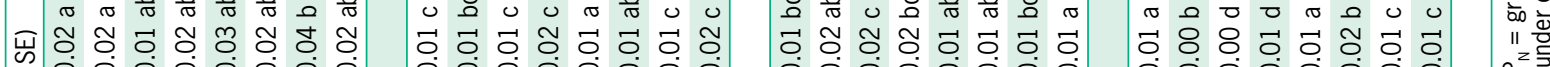

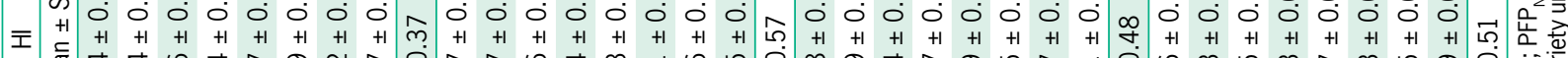

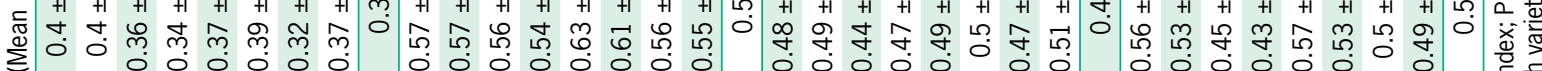

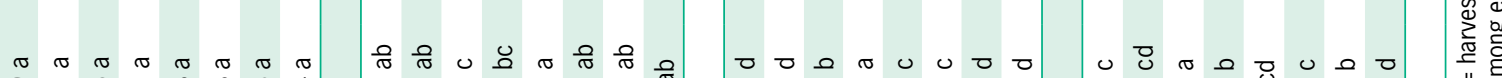

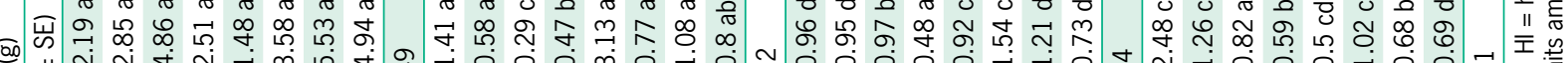

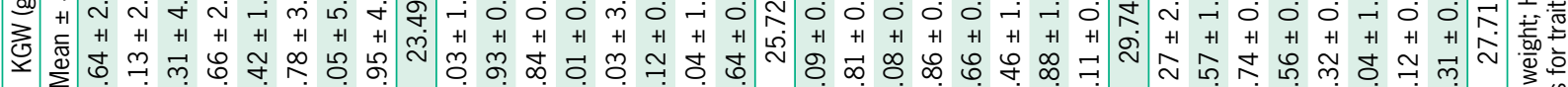
๗ं

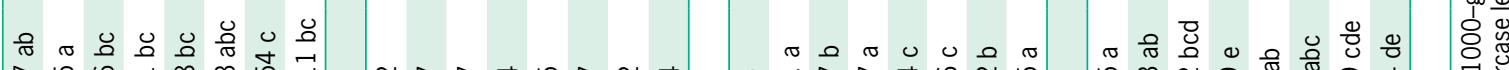

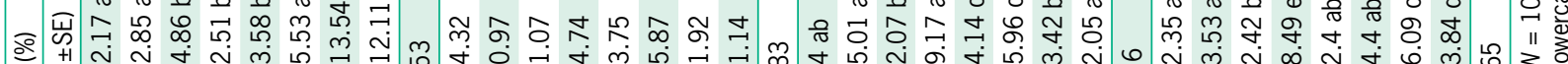

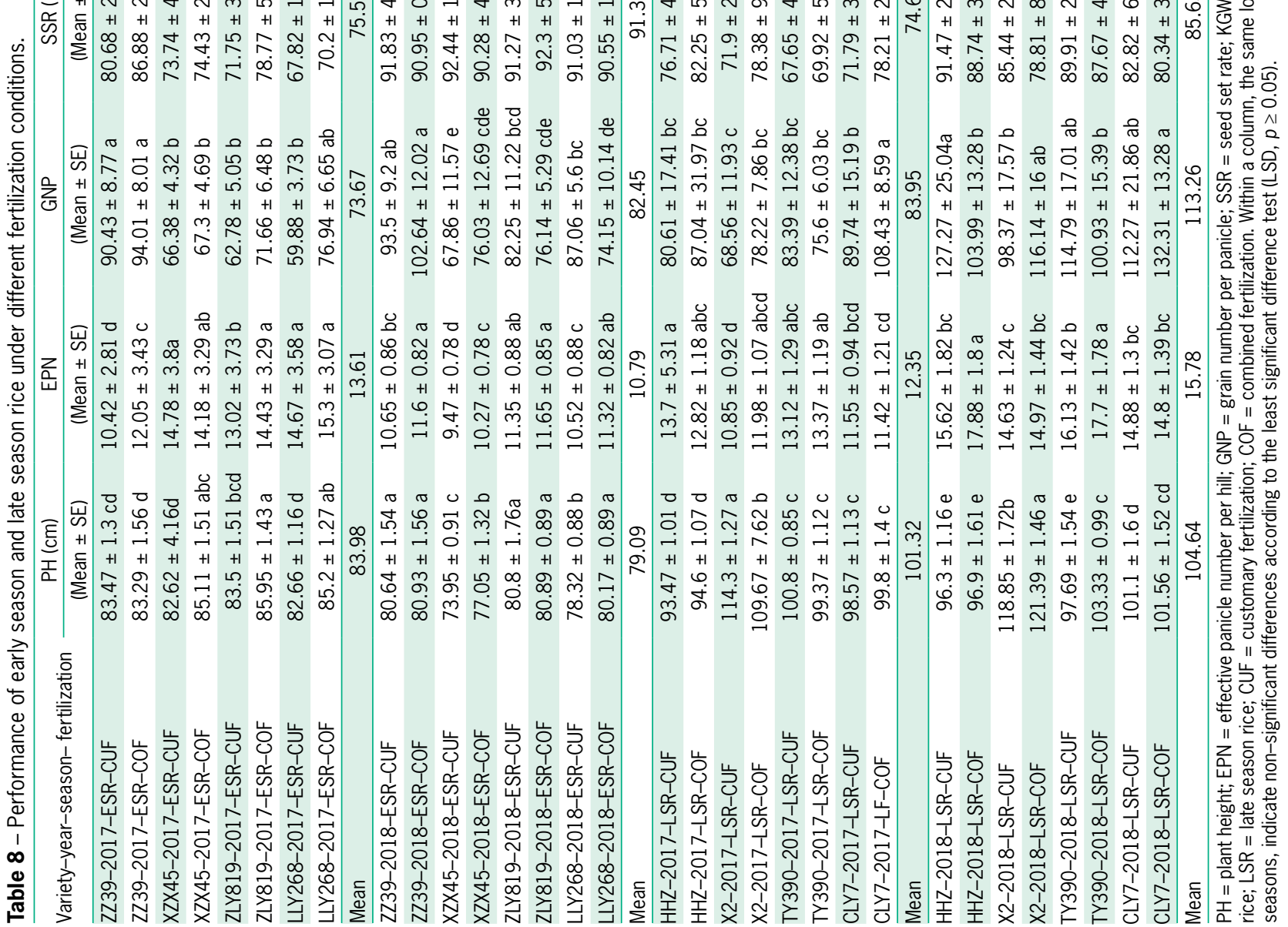


Table 9 - Performance of main traits of different varieties.

\begin{tabular}{lcccccccc}
\hline Year-Season-Fertilization & PH & EPN & GNP & SSR & KGW & HI & Yield $^{\text {PFP }_{N}}$ \\
\hline 2017-ESR-CUF & $\mathrm{cm}$ & & & $\%$ & $\mathrm{~g}$ & & $\mathrm{~kg} \mathrm{~kg}^{-1}$ \\
2017-ESR-COF & 83.06 & 13.22 & 69.87 & 73.50 & 23.36 & 0.36 & 6.59 & 37.82 \\
2018-ESR-CUF & 84.89 & 13.99 & 77.48 & 77.57 & 23.63 & 0.38 & 6.19 & 42.24 \\
2018-ESR-COF & 78.43 & 10.50 & 82.67 & 91.64 & 25.74 & 0.58 & 6.32 & 26.06 \\
2017-LSR-CUF & 79.76 & 11.21 & 82.24 & 91.02 & 25.68 & 0.57 & 7.11 & 38.34 \\
2017-LSR-COF & 101.79 & 12.31 & 80.58 & 72.01 & 29.43 & 0.47 & 8.52 & 51.85 \\
2018-LSR-COF & 100.86 & 12.40 & 87.32 & 77.19 & 30.06 & 0.49 & 8.37 & 60.49 \\
2018-LSR-CUF & 103.49 & 15.32 & 113.18 & 87.41 & 28.55 & 0.52 & 8.90 & 36.73 \\
\hline
\end{tabular}

$\mathrm{ESR}=$ early season rice; $\mathrm{LSR}=$ late season rice; $\mathrm{CUF}=$ customary fertilization; $\mathrm{COF}=$ combined fertilization; $\mathrm{PH}=$ plant height; $\mathrm{EPN}=$ effective panicle number per hill; GNP = grain number per panicle; SSR = seed set rate; KGW = 1000-grain weight; $H \mathrm{H}=$ harvest index; $\mathrm{PFP}_{\mathrm{N}}=$ grain yield with $\mathrm{N}$ application.

Table 10 - Performance of standard heterosis (HCK) for main yield traits of early season and late season rice under different fertilization conditions.

\begin{tabular}{|c|c|c|c|c|c|c|c|c|}
\hline Year-Hybrid-inbred- fertilization & HCK (PH \%) & HCK (EPN \%) & HCK (GNP \%) & HCK (SSR \%) & HCK (KGW \%) & HCK (HI \%) & HCK (Yield \%) & $\mathrm{HCK}\left(\mathrm{PFP}_{\mathrm{N}}\right)$ \\
\hline 2017-ZLY819-ZZ39-CUF & 0.04 & $24.95^{*}$ & $-30.58^{*}$ & -11.07 & -0.93 & -7.5 & 5.4 & 5.39 \\
\hline 2017-ZLY819-ZZ39-COF & $3.19^{*}$ & $19.75^{*}$ & $-23.77^{\star}$ & -9.33 & 7.13 & -2.5 & $16.76^{*}$ & $-7.80^{*}$ \\
\hline 2017-ZLY819-XZX45-CUF & 1.07 & -11.91 & -5.42 & -2.7 & 0.47 & 2.78 & -1.19 & -1.19 \\
\hline 2017-ZLY819-XZX45-COF & 0.99 & 1.76 & 6.48 & 9.78 & 4.73 & 14.71 & $11.97^{*}$ & $-11.57^{*}$ \\
\hline 2017-LLY268-ZZ39-CUF & -0.97 & $40.76^{*}$ & $-33.78^{*}$ & $-15.94^{*}$ & -2.5 & -20 & 6.35 & 6.42 \\
\hline 2017-LLY268-ZZ39-COF & $2.29^{*}$ & $26.97^{*}$ & -18.16 & $-19.2^{*}$ & -0.78 & $-7.5^{*}$ & $20.32^{*}$ & $20.38^{*}$ \\
\hline 2017-LLY268-XZX45-CUF & 0.05 & -0.74 & $-9.79^{*}$ & -8.03 & -1.12 & -11.11 & -0.3 & -1.19 \\
\hline 2017-LLY268-XZX45-COF & 0.11 & 7.9 & 14.32 & -2.16 & -3 & 8.82 & $15.38^{*}$ & $15.45^{*}$ \\
\hline Mean & 0.85 & 13.68 & -12.59 & -7.33 & 0.5 & -2.79 & 9.34 & 3.24 \\
\hline 2018-ZLY819-ZZ39-CUF & 0.2 & 6.57 & -13.68 & -0.61 & 3.84 & $10.53^{*}$ & $17.01^{*}$ & $17.12^{*}$ \\
\hline 2018-ZLY819-ZZ39-COF & -0.05 & 0.43 & $-34.8^{*}$ & 1.48 & 0.73 & 7.02 & $14.09^{*}$ & $14.14^{*}$ \\
\hline 2018-ZLY819-XZZ45-CUF & $9.26^{*}$ & $19.85^{*}$ & $17.5^{*}$ & -1.27 & $13.38^{*}$ & $12.5^{*}$ & $17.41^{*}$ & $17.36^{*}$ \\
\hline 2018-ZLY819-XZX45-COF & $4.98^{*}$ & $13.44^{*}$ & 0.14 & 2.24 & 4.44 & $12.96^{*}$ & $12.76^{*}$ & $12.63^{*}$ \\
\hline 2018-LLY268-ZZ39-COF & $-2.88^{*}$ & -1.22 & -7.4 & -0.87 & 0.04 & -1.75 & 13.1 & 13.12 \\
\hline 2018-LLY268-ZZ39-CUF & -0.94 & -2.41 & $-38.42^{*}$ & -0.44 & -1.12 & -3.51 & 6.53 & 6.46 \\
\hline 2018-LLY268-XZX45-CUF & $5.91^{*}$ & $11.09^{*}$ & $22.05^{*}$ & -1.53 & $9.23^{*}$ & 0 & 13.48 & 13.15 \\
\hline 2018-LLY268-XZX45-COF & $4.05^{\star}$ & $10.22^{*}$ & -2.54 & 0.3 & 2.52 & 1.85 & 5.28 & 5.25 \\
\hline Mean & 2.57 & 7.25 & -7.14 & -0.09 & 4.13 & 4.95 & 12.46 & 12.40 \\
\hline 2017-TY390-HHZ-CUF & $7.84^{*}$ & -4.23 & 3.45 & $-11.81^{*}$ & $9.49^{*}$ & 2.08 & $7.66^{*}$ & 7.69 \\
\hline 2017-TY390-HHZ-COF & $5.04^{*}$ & 4.29 & -13.14 & $-14.99^{*}$ & $5.93^{*}$ & 2.04 & $9.74^{*}$ & $9.68^{*}$ \\
\hline 2017-TY390-X2-CUF & $-11.81^{*}$ & $20.92^{*}$ & 21.63 & $-5.91^{*}$ & $-10.34^{*}$ & $11.36^{*}$ & 3.75 & 3.81 \\
\hline 2017-TY390-X2-COF & $-9.39^{*}$ & 11.6 & -3.35 & $-10.79^{*}$ & $-15.49^{*}$ & 6.38 & $11.93^{*}$ & $11.91^{*}$ \\
\hline 2017-CLY7-HHZ-CUF & $5.46^{*}$ & $-15.69^{*}$ & 11.33 & -6.41 & 2.92 & -2.08 & 3.04 & 2.98 \\
\hline 2017-CLY7-HHZ-COF & $15.93^{*}$ & -10.92 & $24.57^{*}$ & -4.91 & 1.08 & 4.08 & -0.12 & -0.24 \\
\hline 2017-CLY7-X2-CUF & $-13.76^{*}$ & 6.45 & $30.89^{*}$ & -0.15 & $-15.72^{*}$ & 6.82 & -0.7 & -0.73 \\
\hline 2017-CLY7-X2-COF & $-17.79^{*}$ & -4.67 & $38.62^{\circ}$ & -0.22 & $-19.36^{*}$ & $8.51^{*}$ & 1.86 & 1.79 \\
\hline Mean & -0.09 & 0.97 & 14.25 & -6.9 & -5.19 & 4.9 & 4.65 & 4.61 \\
\hline 2018-TY390-HHZ-CUF & 1.44 & 3.27 & -9.81 & -1.71 & -2.52 & 1.79 & 9.15 & 9.14 \\
\hline 2018-TY390-HHZ-COF & $6.64^{*}$ & -1.01 & -2.94 & -1.21 & 1.77 & 0 & 4.19 & 4.12 \\
\hline 2018-TY390-X2-CUF & $-17.8^{*}$ & $10.25^{*}$ & 16.69 & 15.77 & -17.08 & 26.67 & 24.06 & $23.98^{*}$ \\
\hline 2018-TY390-X2-COF & $-14.88^{*}$ & $18.24^{*}$ & -13.1 & 11.24 & -5.32 & 23.26 & 33.9 & $33.79^{*}$ \\
\hline 2018-CLY7-HHZ-CUF & $4.98^{*}$ & -4.74 & -11.79 & -9.46 & 7.85 & -10.71 & -4.63 & -4.6 \\
\hline 2018-CLY7-HHZ-COF & $4.81^{*}$ & $-17.23^{*}$ & 27.23 & -9.47 & -4.74 & -7.55 & -2.1 & -2.16 \\
\hline 2018-CLY7-X2-CUF & $-14.93^{*}$ & 1.71 & 14.13 & -3.07 & -8.25 & 11.11 & 8.4 & 8.38 \\
\hline 2018-CLY7-X2-COF & $-16.34^{*}$ & -1.14 & 13.92 & 1.94 & -11.38 & 13.95 & 25.82 & $25.72^{*}$ \\
\hline Mean & -5.76 & 1.17 & 4.29 & 0.51 & -4.96 & 7.31 & 12.35 & 12.30 \\
\hline
\end{tabular}

$\mathrm{CUF}=$ customary fertilization; $\mathrm{COF}=$ combined fertilization; $\mathrm{PH}=$ plant height; $\mathrm{EPN}=$ effective panicle number per hill; $\mathrm{GNP}=$ grain number per panicle; $\mathrm{SSR}=$ seed set rate; $\mathrm{KGW}=1000$-grain weight; $\mathrm{HI}=$ harvest index; $\mathrm{PFP}_{\mathrm{N}}=$ grain yield with $\mathrm{N}$ application. Within a column in each season and year, ${ }^{*}$ represents a significant difference between the hybrid variety and inbrids variety, according to the least significant difference test (LSD, $p \geq 0.05)$. 
Table 11 - Performance of standard heterosis (HCK)for main traits of early season and late season rice under different fertilization conditions.

\begin{tabular}{lcccccccc}
\hline Year-Season-Fertilization & HCK (PH \%) & HCK (EPN \%) & HCK (GNP \%) & HCK (SSR \%) & HCK (KGW \%) & HCK (HI \%) & HCK (Yield \%) & HCK (PFP \%) \\
\hline 2017-ESR-CUF & 0.05 & 13.27 & -19.89 & -9.44 & -1.02 & -8.96 & 2.57 & 2.36 \\
2017-ESR-COF & 1.65 & 14.10 & -5.28 & -5.23 & 2.02 & 3.38 & 16.11 & 4.12 \\
2018-ESR-CUF & 3.12 & 9.07 & -7.71 & -1.07 & 6.62 & 5.32 & 15.25 & 15.19 \\
2018-ESR-COF & 2.63 & 7.45 & -6.58 & -0.20 & 4.41 & 4.99 & 14.44 & 9.62 \\
2017-LSR-CUF & -3.07 & 1.86 & 16.83 & -6.07 & -3.41 & 4.55 & 3.44 & 3.44 \\
2017-LSR-COF & -1.55 & 0.08 & 11.68 & -7.73 & -6.96 & 5.25 & 5.85 & 5.79 \\
2018-LSR-CUF & -6.58 & 2.62 & 2.31 & 0.38 & -5 & 7.21 & 9.24 & 9.23 \\
2018-LSR-COF & -4.94 & -0.29 & 6.28 & 0.63 & -4.92 & 7.42 & 15.45 & 15.37 \\
\hline
\end{tabular}

$\mathrm{ESR}=$ early season rice; $\mathrm{LSR}=$ late season rice; CUF $=$ customary fertilization; $\mathrm{COF}=$ combined fertilization; $\mathrm{PH}=$ plant height; $\mathrm{EPN}=$ effective panicle number per hill; GNP = grain number per panicle; SSR = seed set rate; KGW = 1000-grain weight; $\mathrm{HI}=$ harvest index; $\mathrm{PFP}_{\mathrm{N}}=$ grain yield with $\mathrm{N}$ application .

early season hybrid rice varieties had a higher SSR under the COF than under the CUF treatment.

In the late season hybrids, the average SSR was $72.01 \%$ and $77.19 \%$ in 2017 and $87.41 \%$ and $83.89 \%$ in 2018 under CUF and COF treatments, respectively (Tables 8 and 9, Figure 4). Compared with late season inbred varieties, the HCK for SSR in late season hybrid rice varieties was $-6.9 \%$ and $0.51 \%$ in 2017 and -6.07 $\%$ and $-7.73 \%$ in 2018 under CUF and COF treatments, respectively (Tables 10 and 11, Figure 5). These results showed that SSR was not the main yield heterosis trait in either early or late season hybrid rice. Notably, the heterosis in SSR was unaffected by fertilizer treatments.

Several previous studies have shown that increasing the spikelet size (grain weight) is a feasible approach to increasing rice yield (Huang et al., 2011; Zhang et al., 2009). To measure this trait, we examined 1000-grain weight (KGW) and compared the trait between varieties and across fertilizer treatments to determine if lower fertilization negatively impacted yield. The KGW of early season rice was 23.36 and $23.63 \mathrm{~g}$ in 2017 and 25.74 and $25.68 \mathrm{~g}$ in 2018 under CUF and COF treatments, respectively (Tables 8 and 9, Figure 4). Compared with early season inbred varieties, the average $\mathrm{KGW}$ of early season hybrid rice varieties was $-1.02 \%$ and $2.02 \%$ higher in 2017 and $6.62 \%$ and $4.41 \%$ higher in 2018 under CUF and COF treatments, respectively (Tables 10 and 11, Figure 5). Thus, the early season hybrid rice varieties exhibited an advantage in terms of KGW as compared to the early season inbred rice varieties.

The KGW of late season rice was 29.43 and $30.06 \mathrm{~g}$ in 2017 and 28.55 and $26.87 \mathrm{~g}$ in 2018 under CUF and COF treatments, respectively (Tables 8 and 9, Figure 4). However, in comparison with the late season inbred varieties, the HCK for KGW of late season hybrid rice varieties was $-3.41 \%$ and $-6.96 \%$ in 2017 and $-5 \%$ and $-4.92 \%$ in 2018 under CUF and COF treatments, respectively, thus exhibiting poor heterosis for KGW, regardless of fertilizer treatment (Tables 10 and 11, Figure 5). These data showed that, in terms of $\mathrm{KGW}$, differences in fertilizer treatment did not lead to significant improvement in the yield of hybrid rice over that of inbred rice.

\section{Decreasing fertilizer inputs did not affect $\mathrm{HI}$, grain yield, or NUE for either early or late season hybrids}

The degree of source-to-sink translocation is often assessed by measuring the harvest index (Sinclair, 1998), which is determined by the rate of transient photosynthesis during grain formation and the remobilization of stored reserves into the growing grain (Blum, 1993). It is generally accepted that the HI (harvest index) of modern high-yielding rice cultivars is approximately 0.5 (Zhong et al., 2006). Herein, we found that the HI of early season rice was $0.32-0.63$, with HI values being 0.36 and 0.38 for CUF and COF treatments, respectively, in 2017, and 0.58 and 0.57 for CUF and COF treatments, respectively, in 2018 (Tables 8 and 9, Figure 4). Thus, the difference in HI under fertilization conditions was little, and it was greater between both varieties and years. Specifically, compared with the early season inbred varieties, the average HCK for HI in early season hybrids was $-8.96 \%$ and $3.38 \%$ in 2017 and $5.32 \%$ and $4.99 \%$ in 2018 under CUF and COF treatments, respectively (Tables 10 and 11, Figure 5).

In contrast, the $\mathrm{HI}$ for late season rice was $0.43-$ 0.57 , with $\mathrm{HI}$ values being 0.47 and 0.49 for CUF and COF treatments, respectively, in 2017, and 0.52 and 0.50 for CUF and COF treatments, respectively, in 2018 (Tables 8 and 9, Figure 4). Similarly, the differences were subtle under the fertilization conditions, but significant between rice varieties. In the comparison of heterosis in late season inbred varieties, the HCK for HI of late season hybrid rice varieties was higher than that of inbred varieties by $4.55 \%$ and $7.21 \%$ for CUF and COF treatments, respectively, in 2017 and by $5.25 \%$ and $7.42 \%$ for the respective treatments in 2018. Thus, there was positive heterosis for this trait (Tables 10 and 11, Figure 5). In the case of $\mathrm{HI}$, late hybrid rice performed better under the low fertilizer COF treatment than under the CUF treatment.

The final metric for performance was yield, and we found that early hybrid varieties yielded between 5.61-7.69 $\mathrm{t} \mathrm{ha}^{-1}$ (tons per hectare). The average yield of early season rice was 6.59 and $6.19 \mathrm{t} \mathrm{ha}^{-1}$ in 2017 and 6.32 and $7.11 \mathrm{t} \mathrm{ha}^{-1}$ in 2018 under CUF and COF 
treatments, respectively (Tables 8 and 9, Figure 4). The average HCK for yield among early season hybrids was higher by $2.57 \%$ and $16.11 \%$ than that of inbred lines in 2017 and by $15.25 \%$ and $14.44 \%$ than that of inbred lines in 2018 under CUF and COF conditions, respectively (Tables 10 and 11, Figure 5). It reflected a better performance by early season hybrids than that of the inbred varieties, under COF conditions.

The yield for late season rice ranged from 7.05 to $9.9 \mathrm{t} \mathrm{ha}^{-1}$. The yield of late season rice was 8.52 and 8.37 t ha $\mathrm{h}^{-1}$ in 2017 and 8.90 and $8.61 \mathrm{t} \mathrm{ha}^{-1}$ in 2018 under CUF and COF conditions, respectively (Tables 8 and 9, Figure 4). The average HCK for yield of late season hybrid varieties was $3.44 \%$ and $5.85 \%$ higher than that of inbred lines in 2017, and $9.24 \%$ and $15.45 \%$ higher than that of inbred lines in 2018 under CUF and COF treatments, respectively (Tables 10 and 11, Figure $5)$. Both the early and late season hybrid varieties had higher yields than that of the inbred lines, and yields of hybrids were more stable and consistently higher under the COF treatment than under the CUF treatment.

$\mathrm{PFP}_{\mathrm{N}}$ (kg rice grain per $\mathrm{kg} \mathrm{N}$ applied) is used as the broadest measure of NUE. The average $\mathrm{PFP}_{\mathrm{N}}$ of early season rice was 37.82 and $42.24 \mathrm{~kg} \mathrm{~kg}^{-1}$ in 2017 and 26.06 and $38.34 \mathrm{~kg} \mathrm{~kg}^{-1}$ in 2018 under CUF and COF conditions, respectively (Tables 8 and 9, Figure 4). The average $\mathrm{HCK}$ for $\mathrm{PFP}_{\mathrm{N}}$ of early season hybrids was $2.36 \%$ and $4.12 \%$ higher than that of the inbred lines in 2017, and $15.19 \%$ and $9.62 \%$ higher than that of inbred lines in 2018 under CUF and COF conditions, respectively (Tables 10 and 11, Figure 5).

The average $\mathrm{PFP}_{\mathrm{N}}$ of late season rice was 51.85 and $60.49 \mathrm{~kg} \mathrm{~kg}^{-1}$ in 2017 and 36.73 and $49.14 \mathrm{~kg} \mathrm{~kg}^{-1}$ in 2018 under CUF and COF conditions, respectively (Tables 8 and 9, Figure 4). The average HCK for PFP ${ }_{N}$ of early season hybrids was $3.44 \%$ and $5.79 \%$ higher than that of the inbred lines in 2017, and $9.23 \%$ and $15.37 \%$ higher than that of inbred lines in 2018 under CUF and COF conditions, respectively (Tables 10 and 11, Figure 5). Both the early and late season hybrid varieties had higher $\mathrm{PFP}_{\mathrm{N}}$ than that of the inbred lines, and the $\mathrm{PFP}_{\mathrm{N}}$ values were higher under the COF treatment than under the CUF treatment, except for early season 2018. Most of the $\mathrm{PFP}_{\mathrm{N}}$ of early and late season hybrid varieties corresponded to their yields under the COF treatment rather than the CUF treatment.

\section{Discussion}

Source capacity is usually expressed as the amount of biomass production, which is achieved through the plant's photosynthetic capacities. Sink capacity is a function of the number of spikelets per unit land area and their potential size. Source-to-sink translocation degree is often assessed by measuring the harvest index, which is determined by the transient photosynthesis during grain formation and the remobilization of stored reserves into the growing grain (Blum, 1993; Mae et al.,
2006; Sinclair, 1998; Zhang et al., 2009). Hybrid rice yielded approximately 7-19\% more than that of inbred cultivars. The higher grain yields observed for hybrid rice cultivars were attributed to high grain weight and biomass accumulation, with longer crop duration to make full use of the heat/light resources in the growing season (Bueno and Lafarge, 2009; Jiang et al., 2016; Katsura et al., 2007; Yang et al., 2007). The attainable early season rice yield under double rice cropping systems is characterized by a relatively lower grain yield of 5-6 $\mathrm{t} \mathrm{ha}^{-1}$ and superiority in sink size (sink capacity, such as spikelets per $\mathrm{m}^{2}$ ) and biomass production (Wu et al., 2013; Zhong et al., 2006). A recent investigation by Chen et al., 2019 showed that the highest yield of early season hybrid rice was $7.60 \mathrm{t} \mathrm{ha}^{-1}$ and $7.49 \mathrm{t} \mathrm{ha}^{-1}$ in 2017 and 2018, respectively, with the yield advantage of early season hybrids typically being less than $5 \%$ over that of inbred varieties; furthermore, hybrids were shown to produce more panicles per plant but less grains per panicle (Chen et al., 2019). In this study, it was revealed that the average yield of early season rice was $6.39 \mathrm{t} \mathrm{ha}^{-1}$ and $6.71 \mathrm{t} \mathrm{ha}^{-1}$ in 2017 and 2018, respectively (Table 8). Compared with inbred lines, the average yield of early season hybrid rice increased by $9.34 \%$ and $12.46 \%$ in 2017 and 2018, respectively (Table 10). Early season rice hybrids had leading advantages in terms of EPN, KGW, HI, Yield, and NUE (Figure 6A and 6B), with the EPN advantage being hugely evident for them, though they were affected seriously by year, variety and fertilization (Table 12). The results of this study were consistent with those reported by Chen et al. (2019). However, in our study, the yield advantage of early season hybrid rice was greater and not limited as compared to inbred rice.

A previous study evinced that GNP and EPN traits were the main causes for yield superiority in the two line super high-yielding hybrid rice varieties with long growth duration. The GNP and EPN had super-parental advantages (Li et al., 2016). The highest yield of late season hybrid rice was $9.64 \mathrm{t} \mathrm{ha}^{-1}$, which produced a 6-26\% higher grain yield than that of the other cultivars because the higher grain yield was driven by improvements in sink-source capacity (biomass production, panicles and spikelets per $\mathrm{m}^{2}$, and grain weight) (Huang et al., 2017b). The study showed that the average yield of late season rice was $8.44 \mathrm{t} \mathrm{ha}^{-1}$ and $8.75 \mathrm{t} \mathrm{ha}^{-1}$ in 2017 and 2018, respectively (Table 8). Compared with the inbred lines, the average yield of late season hybrid rice increased by $4.65 \%$ and 12.35 $\%$ in 2017 and 2018, respectively (Table 10). The late season hybrid rice had obvious advantages in GNP, HI, yield, and NUE (Figure 6C and 6D). The GNP was an obvious advantage for late season hybrid rice, different from early season hybrid rice. This result is similar to that reported by $\mathrm{Li}$ et al. (2016).

Nitrogen $(N)$ is the most important nutrient element in irrigated rice production, and current high yields of irrigated rice are associated with large applications of fertilizer (Cassman et al., 1998). To fulfill 

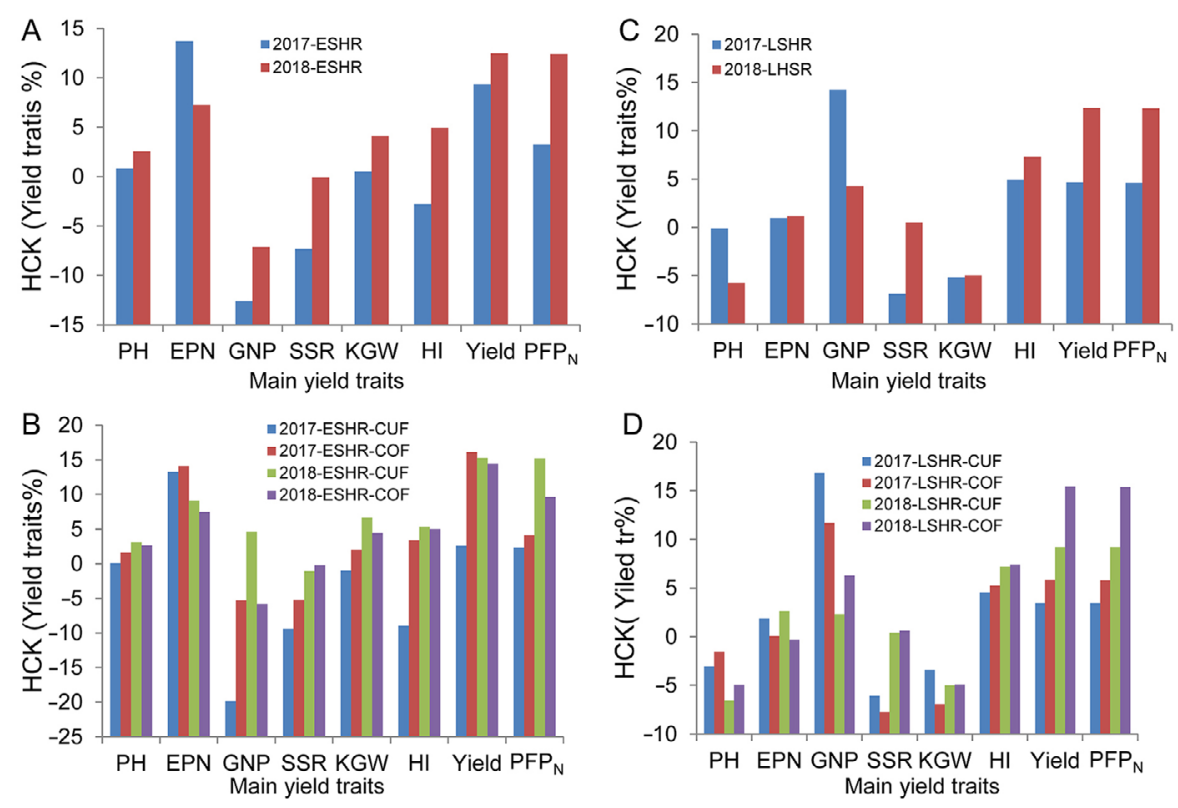

Figure 6 - Standard heterosis (HCK) for early season and late season hybrid rice under different fertilization conditions. PH = Plant height; EPN = effective panicle number; GNP = grain number per panicle; $\mathrm{SSR}=$ seed set rate; $\mathrm{KGW}=1000$ grain weight; $\mathrm{HI}=$ harvest index; $P F P_{\mathrm{N}}=\mathrm{N}$ partial factor productivity; ESHR = early season hybrid rice; LSHR = late season hybrid rice; CUF = customary fertilization; COF = combined fertilization.

Table 12 - Analysis of variance showing the significance probability of each effects for different agronomic traits.

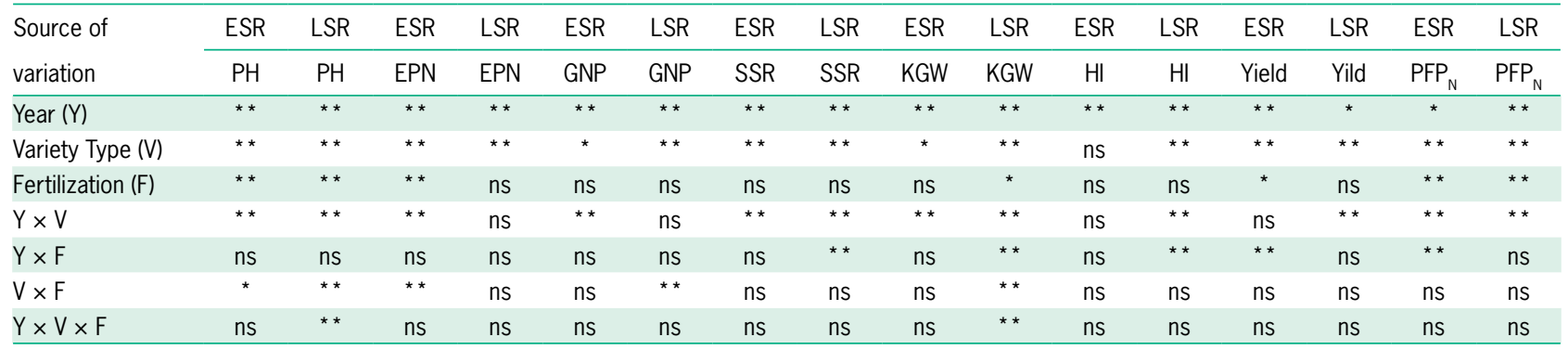

ESR = early season rice; $L S R=$ late season rice; $C U F=$ customary fertilization; $C O F=$ combined fertilization; $P H=$ plant height; $E P N=$ effective panicle number per

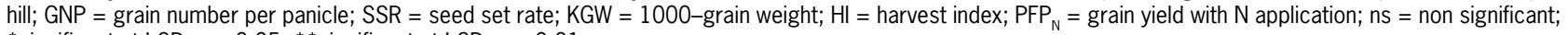
* significant at LSD, $p \geq 0.05 ;{ }^{* *}$ significant at LSD, $p \geq 0.01$.

the yield potential of the super hybrid rice, $\mathrm{N}$ input of more than $350 \mathrm{~kg} \mathrm{~N}^{-1}$ was needed (Wang and Peng, 2017). The yield of hybrid rice increased by $59-71 \%$ when the $\mathrm{N}$ application rate increased from 150 to 210 $\mathrm{kg} \mathrm{ha}^{-1}$. The results of grain yield, NUE, and apparent $\mathrm{N}$ balance (ANB) indicated that the $180 \mathrm{~kg} \mathrm{ha}^{-1}$ rate of $\mathrm{N}$ application was most effective (Yousaf et al., 2016). The average yield of hybrid cultivars LYPJ and YLY1 was $22 \%$ and $16 \%$ higher than that of inbred cultivars HHZ and YXYZ. The higher grain yield with $\mathrm{N}$ fertilizer in hybrid rice was driven more by a higher yield without $\mathrm{N}$ fertilizer than by increases in grain yield with $\mathrm{N}$ fertilizer under moderate to high soil fertility conditions (Huang et al., 2017a). The grain yield of super hybrid rice was higher than that of inbred rice by 3.33-7.41\% at N0 10 $\mathrm{kg} \mathrm{N} \mathrm{ha}{ }^{-1}$ ) and 5.94-19.87\% at N90 (90 kg N ha-1) (Huang et al., 2018). Another study demonstrated that N2 (125$176 \mathrm{~kg} \mathrm{~N} \mathrm{ha}^{-1}$ ) had higher agronomic NUE, whereas the difference in grain yield between N1 (225 kg N ha-1) and N2 (125-176 kg N ha-1) was relatively slight (Jiang et al., 2016). In this study, the total $\mathrm{N}$ fertilizer application of early season rice was 174.17 and $138.34 \mathrm{~kg} \mathrm{ha}^{-1}$ in 2017 and 242.32 and $185.34 \mathrm{~kg} \mathrm{ha}^{-1}$ in 2018 under conditions of CUF and COF respectively; the average yield of early season rice was 6.59 and $6.19 \mathrm{t} \mathrm{ha}^{-1}$ in 2017 and 6.32 and $7.11 \mathrm{t} \mathrm{ha}^{-1}$ in 2018 under conditions of CUF and $\mathrm{COF}$, respectively. Compared with the inbred varieties, the HCK for yield of early season hybrid rice was $2.57 \%$ and $16.11 \%$ higher than that of inbred varieties in 2017 and $15.25 \%$ and $14.44 \%$ higher than that of inbred varieties in 2018 under conditions of CUF and COF, respectively. The total $\mathrm{N}$ fertilizer application of late 
season rice was 164.25 and $138.34 \mathrm{~kg} \mathrm{ha}^{-1}$ in 2017 and 242.32 and $175.1 \mathrm{~kg} \mathrm{ha}^{-1}$ in 2018 under conditions of CUF and COF, respectively. Compared with the inbred varieties, the HCK of late season hybrid rice was 3.44 $\%$ and $5.85 \%$ in 2017 and $9.24 \%$ and $15.45 \%$ in 2018 under conditions of CUF and COF, respectively. The yield of late season rice was 8.52 and $8.37 \mathrm{t} \mathrm{ha}^{-1}$ in 2017 , and 8.90 and $8.61 \mathrm{tha}^{-1}$ in 2018 under conditions of CUF

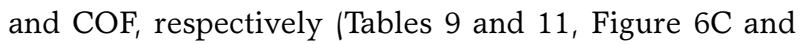
$6 \mathrm{D})$. It denoted that the yield advantage of hybrid rice under COF was higher than that under CUF.

Macronutrients $\mathrm{N}, \mathrm{P}$ and $\mathrm{K}$ are the primary nutrients and fundamental to crop development because of their being the basic components of many organic molecules, nucleic acids and proteins. Most modern rice cultivars are bred under large chemical application of fertilizer (Lea and Miflin, 2011). Soil organic matter (SOM) retains and recycles nutrients, improves soil physical structure, and impacts crop growth. Soil organic carbon (SOC) storage is the key function of agricultural soil, as it interacts with other functions, e.g., soil fertility, nutrient cycling, temperature, and $\mathrm{pH}$ balance (Lal et al., 2007; Six et al., 2002). It is also related to sustainable strategies to mitigate the emission of greenhouse gases (GHGs) (Arunrat and Pumijumnong, 2017; Rajan et al., 2017). Increasing SOC content by $1 \mathrm{~g} \mathrm{~kg}^{-1}$ can increase rice yield by $302 \mathrm{~kg} \mathrm{ha}^{-1}$ (Arunrat et al., 2020a). Via many processes of an oxidation-reduction reaction, SOC dynamics change soil $\mathrm{pH}$, reduction of $\mathrm{C}$, nitrogen $(\mathrm{N})$, sulfur (S), and redox potential in a waterlogged rice field (Fageria et al., 2011). The secondary nutrients include calcium (Ca), magnesium (Mg) and sulfur (S). These three are needed in lesser amounts than the primary nutrients. The micronutrients are boron $(\mathrm{B})$, chlorine $(\mathrm{Cl})$, copper $(\mathrm{Cu})$, iron $(\mathrm{Fe})$, manganese $(\mathrm{Mn})$, molybdenum $(\mathrm{Mo})$, and zinc $(\mathrm{Zn})$. These elements are used in very small amounts, but they are just as important to plant development and profitable crop production (Ren et al., 2007; Sakai et al., 2015; Xia et al., 2016; Zhang et al., 2018).

Farmers in China usually over-apply synthetic N fertilizer to maximize grain yield, resulting in a steep decline in NUE (Ju et al., 2009; Miao et al., 2011). A PFP of $41.1 \mathrm{~kg} \mathrm{~kg}^{-1}$ of irrigated rice was reported previously on a national scale in China (Xu et al., 2018). $\mathrm{PFP}_{\mathrm{N}}$ values of $50 \mathrm{~kg} \mathrm{~kg}^{-1}$ and above are generally considered to be achievable with good management (Dobermann and Fairhurst, 2000; Xie et al., 2020). In this study, the $\mathrm{PFP}_{\mathrm{N}} \mathrm{s}$ of early season rice were 37.82 and $42.24 \mathrm{~kg} \mathrm{~kg}^{-1}$ in 2017 , and 26.06 and $38.34 \mathrm{~kg} \mathrm{~kg}^{-1}$ in 2018 under CUF and COF treatments, respectively, whereas the $\mathrm{PFP}_{\mathrm{N}} \mathrm{s}$ of late season rice were 51.85 and $60.49 \mathrm{~kg} \mathrm{~kg}^{-1}$ in 2017 , and 36.73 and $49.14 \mathrm{~kg} \mathrm{~kg}^{-1}$ in 2018 under CUF and COF treatments, respectively. NUE under COF treatment was much higher than that under CUF treatment. Recent research shows that the double activation of OsAMT1;2 and OsGOGAT1 increases plant performance in agriculture, providing better $\mathrm{N}$ grain filling without yield penalty under paddy field conditions, as well as better grain yield and $\mathrm{N}$ content when plants are grown under $\mathrm{N}$ limitations in field conditions (Chen, 2013). It provides a recommended breeding strategy for improving plant growth, NUE, and yield advantage of hybrid rice in future.

Previous studies have suggested that small farm and smallholder management are key causes of low agricultural productivity worldwide (Ju et al., 2016). In China, the rice planting area per household was on an average of $0.33 \mathrm{ha}$, and $60 \%$ of farmers had $<0.3$ ha of planting area (Xie et al., 2020). Most farmers lacked the knowledge of required application of $\mathrm{N}$-fertilizer to obtain optimal amounts of grain production. Consequently, they supposed that applying more $\mathrm{N}$, regardless of how much a crop needed, was an "insurance" strategy against low yields (Jiao et al., 2018; Vitousek et al., 2009; Zhang and Yang, 2016). Combined fertilization can reduce $\mathrm{N}$ fertilizer and the total fertilizer application, whilst achieving high yield, and further improving NUE when used on hybrid rice, while being convenient for elderly farmers to use against weeds and insect pests.

\section{Conclusions}

EPN, GNP, SSR, and KGW are very important rice yield traits. The EPN was the prime advantage of early season hybrid rice with a short growth period, where as the GNP was the main advantage of late season hybrid rice with a long growth period. The main yield characteristic advantage of hybrid rice was stronger under combined (low) fertilization than under customary (high) fertilization. In summary, high yield can be achieved by selecting excellent hybrid rice varieties and using combined fertilization (low fertilizer). Combined fertilization can be more profitable for rice growers due to reduced use of fertilizer and pesticides, which also means leading to less labor and other production costs.

\section{Acknowledgment}

This work was the Key Research and Development Program of China (Grant number 2017YFD0301504). This article is dedicated to respected Professor Longping Yuan.

\section{Authors' Contributions}

Conceptualization: $\mathrm{Zhu}$, L.H; Yuan, L.P. Data acquisition: $\mathrm{Wu}, \mathrm{Z} . \mathrm{H}$; Peng, Y.L.; Tan, F.J. Design of methodology: Xin, Y.Y; Lv, Q.M. Writing and editing: Huang, Z.Y; Hossain, M.A.

\section{References}

Ao, H.; Peng, S.; Zou, Y.; Tang, Q.; Visperas, R.M. 2010. Reduction of unproductive tillers did not increase the grain yield of irrigated rice. Field Crops Research 116: 108-115. https://doi. org/10.1016/j.fcr.2009.11.020 
Arunrat, N.; Kongsurakan, P.; Sereenonchai, S.; Hatano, R. 2020a. Soil organic carbon in sandy paddy fields of northeast Thailand: a review. Agronomy 10: 1061. https:// doi.org/10.3390/agronomy 10081061

Arunrat, N.; Pumijumnong, N. 2017. Practices for reducing greenhouse gas emissions from rice production in northeast Thailand. Agriculture 7: 4. https://doi.org/10.3390/ agriculture 7010004

Arunrat, N.; Pumijumnong, N.; Sereenonchai, S.; Chareonwong, U.; Wang, C. 2020b. Assessment of climate change impact on rice yield and water footprint of largescale and individual farming in Thailand. Science of The Total Environment 726: 137864. https://doi.org/10.1016/j. scitotenv.2020.137864

Arunrat, N.; Pumijumnong, N.; Sereenonchai, S.; Chareonwong, U.; Wang, C. 2020c. Comparison of GHG emissions and farmers' profit of large scale and individual farming in rice production across four regions of Thailand. Journal of Cleaner Production 278: 123945. https://doi.org/10.1016/j. jclepro.2020.123945

Blum, A. 1993. Selection for sustained production in waterdeficit environments. p. 347. In: Buxton, D.R.; Shibles, R.A.; Forsberg, R.A.; Blad, B.L, eds. International crop science. Crop Science Society of America, Madison, WI, USA.

Bueno, C.S.; Lafarge, T. 2009. Higher crop performance of rice hybrids than of elite inbreds in the tropics. 1. Hybrids accumulate more biomass during each phenological phase. Field Crops Research 112: 229-237. https://doi.org/10.1016/j. fcr.2009.03.006

Cai, H.; Chen, Q. 2000. Rice research in China in the early $21^{\text {st. }}$ century. Chinese Rice Research Newsletter 8: 14-16.

Carpenter, S.R. 2008. Phosphorus control is critical to mitigating eutrophication. Proceedings of the National Academy of Sciences of the United States of America 105: 11039-11040. https://doi.org/10.1073/pnas.0806112105

Cassman, K.G.; Gines, G.C.; Dizon, M.A.; Samson, M.I.; Alcantara, J.M. 1996. Nitrogen-use efficiency in tropical lowland rice systems: contributions from indigenous and applied nitrogen. Field Crops Research 47: 1-12. https:// doi.org/10.1016/0378-4290(95)00101-8

Cassman, K.G.; Peng, S.; Olk, D.; Ladha, J.K.; Reichardt, W.; Dobermann, A.; Singh, U. 1998. Opportunities for increased nitrogen-use efficiency from improved resource management in irrigated rice systems. Field Crops Research 56: 7-39. https://doi.org/10.1016/S0378-4290(97)00140-8

Chen, J.; Du, C.; Zhang, H.; Dai, D.; Ma, L. 2019. Limited yield advantage of early-season rice hybrids over inbreds in middle-lower reaches of the Yangtze River. Molecular Breeding 39: 107. https://doi.org/10.1007/s11032-0191018-z

Chen, Z.J. 2013. Genomic and epigenetic insights into the molecular bases of heterosis. Nature Reviews Genetics 14: 471. https://doi.org/10.1038/nrg3503

Dobermann, A.; Fairhurst, T. 2000. Rice: Nutrient Disorders and Nutrient Management. IRRI: Manila, Philippines.

Fageria, N.K.; Baligar, V.C. 2005. Enhancing nitrogen use efficiency in crop plants. Adv. Agron. 88: 97-185. https:// doi.org/10.1016/S0065-2113(05)88004-6
Fageria, N.K.; Carvalho, G.D.; Santos, A.; Ferreira, E.P.B.; Knupp, A.M. 2011. Chemistry of lowland rice soils and nutrient availability. Communications in Soil Science and Plant Analysis 42: 1913-1933. https://doi.org/10.1080/0010 3624.2011.591467

Huang, L.; Sun, F.; Yuan, S.; Peng, S.; Wang, F. 2018. Different mechanisms underlying the yield advantage of ordinary hybrid and super hybrid rice over inbred rice under low and moderate $\mathrm{N}$ input conditions. Field Crops Research 216: 150-157. https://doi.org/10.1016/j.fcr.2017.11.019

Huang, M.; Jiang, P.; Shan, S.; Gao, W.; Ma, G.; Zou, Y.; Uphoff, N.; Yuan, L. 2017a. Higher yields of hybrid rice do not depend on nitrogen fertilization under moderate to high soil fertility conditions. Rice 10: 43-43. https://doi.org/10.1186/s12284-0170182-1

Huang, M.; Shan, S.; Zhou, X.; Chen, J.; Cao, F.; Jiang, L.; Zou, Y. 2017b. Agronomic performance of late-season rice in south China. Plant Production Science 21: 32-38.

https://doi.org/10.1080/1343943X.2017.1418629

Huang, M.; Zou, Y.; Jiang, P.; Xia, B.; Ibrahim, A.H. 2011. Relationship between grain yield and yield components in super hybrid rice. Agricultural Sciences in China 10: 15371544. https://doi.org/10.1016/S1671-2927(11)60149-1

Jiang, P.; Xie, X.; Huang, M.; Zhou, X.; Zhang, R.; Chen, J.; Wu, D.; Xia, B.; Xiong, H.; Xu, F. 2016. Potential yield increase of hybrid rice at five locations in southern China. Rice 9: 11-11. https://doi.org/10.1186/s12284-016-0085-6

Jiao, X.; Mongol, N.; Zhang, F.S. 2018. The transformation of agriculture in China: looking back and looking forward. Journal of Integrative Agriculture 17: 755-764. https://doi.org/10.1016/ S2095 755-764. -3119(17)61774-X

Ju, X.; Gu, B.; Wu, Y.; Galloway, J.N. 2016. Reducing China's fertilizer use by increasing farm size. Global Environmental Change-human and Policy Dimensions 41: 26-32. https://doi. org/10.1016/j.gloenvcha.2016.08.005

Ju, X.; Xing, G.; Chen, X.; Zhang, S.; Zhang, L.; Liu, X.; Cui, Z.; Yin, B.; Christie, P.; Zhu, Z. 2009. Reducing environmental risk by improving $\mathrm{N}$ management in intensive Chinese agricultural systems. Proceedings of the National Academy of Sciences of the United States of America 106: 3041-3046. https://doi. org/10.1073/pnas.0813417106

Katsura, K.; Maeda, S.; Horie, T.; Shiraiwa, T. 2007. Analysis of yield attributes and crop physiological traits of Liangyoupeijiu, a hybrid rice recently bred in China. Field Crops Research 103: 170-177. https://doi.org/10.1016/j.fcr.2007.06.001

Lal, R.; Follett, R.F.; Stewart, B.A.; Kimble, J.M. 2007. Soil carbon sequenstration to migitigate climate change and advance food security. Soil Science 172: 943-956. https://doi.org/10.1097/ ss.0b013e 31815 cc498

Lea, P.J.; Miflin, B.J. 2011. Nitrogen Assimilation and its Relevance to Crop Improvement. Wiley-Blackwell, London, UK.

Li, D.; Huang, Z.; Song, S.; Xin, Y.; Mao, D.; Lv, Q.; Zhou, M.; Tian, D.; Tang, M.; Wu, Q. 2016. Integrated analysis of phenome, genome, and transcriptome of hybrid rice uncovered multiple heterosis-related loci for yield increase. Proceedings of the National Academy of Sciences of the United States of America 113: E6026. https://doi.org/10.1073/ pnas. 1610115113 
Li, G. 2018. Research progress of medicine fertilizer. Henan Chemical Industry 35: 12-15. https://doi: 10.14173/j.cnki. hnhg.2018.07.003 (in Chinese, with abstract in English).

Liu, X.; Zhang, Y.; Han, W.; Tang, A.; Shen, J.; Cui, Z.; Vitousek, P.M.; Erisman, J.W.; Goulding, K.W.T.; Christie, P. 2013. Enhanced nitrogen deposition over China. Nature 494: 459-462. https://doi.org/10.1038/ nature 11917

Mae, T.; Inaba, A.; Kaneta, Y.; Masaki, S.; Sasaki, M.; Aizawa, M.; Okawa, S.; Hasegawa, S.; Makino, A. 2006. A largegrain rice cultivar, Akita 63, exhibits high yields with high physiological N-use efficiency. Field Crops Research 97: 227-237. https://doi.org/10.1016/j.fcr.2005.10.003

Miao, Y.; Stewart, B.A.; Zhang, F. 2011. Long-term experiments for sustainable nutrient management in China: a review. Agronomy for Sustainable Development 31: 397-414. https://doi.org/10.1051/agro/2010034

Miller, B.C.; Hill, J.E.; Roberts, S.R. 1991. Plant population effects on growth and yield in water-seeded rice. Agronomy Journal 83: 291-297.

Neumann, K.; Verburg, P.H.; Stehfest, E.; Muller, C. 2010. The yield gap of global grain production: a spatial analysis. Agricultural Systems 103: 316-326. https://doi. org/10.1016/j.agsy.2010.02.004

Norse, D.; Ju, X. 2015. Environmental costs of China's food security. Agriculture, Ecosystems \& Environment 209: 5-14. https://doi.org/10.1016/j.agee.2015.02.014

Peng, S.; Huang, J.; Sheehy, J.E.; Laza, R.C.; Visperas, R.M.; Zhong, X.; Centeno, G.S.; Khush, G.S.; Cassman, K.G. 2004. Rice yields decline with higher night temperature from global warming. Proceedings of the National Academy of Sciences of the United States of America 101: 9971-9975. https://doi.org/10.1073/pnas.0403720101

Peng, S.; Tang, Q.; Zou, Y. 2009. current status and challenges of rice production in China. Plant Production Science 12: 3-8. https://doi.org/10.1626/pps.12.3

Rajan, G.; Sushil, L.; Acharya, B.S.; Bista, P.; Sainju, U.M. 2017. Tillage, crop residue, and nutrient management effects on soil organic carbon in rice-based cropping systems: a review. Journal of Integrative Agriculture 16: 1-15. https://doi.org/10.1016/S2095-3119(16)61337-0

Ray, D.K.; Foley, J.A. 2013. Increasing global crop harvest frequency: recent trends and future directions. Environmental Research Letters 8: 044041. http://dx.doi. org/10.1088/1748-9326/8/4/044041

Ren, X.L.; Liu, Q.L.; Fu, H.W.; Wu, D.X.; Shu, Q.Y. 2007. Density alteration of nutrient elements in rice grains of a low phytate mutant. Food Chemistry 102: 1400-1406. https://doi.org/10.1016/j.foodchem.2006.05.065

Sakai, H.; Iwai, T.; Matsubara, C.; Usui, Y.; Okamura, M.; Yatou, O.; Terada, Y.; Aoki, N.; Nishida, S.; Yoshida, K.T. 2015. A decrease in phytic acid content substantially affects the distribution of mineral elements within rice seeds. Plant Science 238: 170-177. https://doi.org/10.1016/j. plantsci.2015.06.006

Sinclair, T.R. 1998. Historical changes in harvest index and crop nitrogen accumulation. Crop Science 38: 638-643. https://doi. org/10.2135/cropsci1998.0011183X003800030002x|
Six, J.; Conant, R.T.; Paul, E.A.; Paustian. K. 2002. Stabilization mechanisms of soil organic matter: implications for C-saturation of soils. Plant \& Soil 241: 155-176. https://doi.org/10.1023/A:1016125726789

van Noordwijk, M.; Brussaard, L. 2014. Minimizing the ecological footprint of food: Closing yield and efficiency gaps simultaneously? Curr. Opin. Environ. Sustain. 8: 62-70. https://doi.org/10.1016/j. cosust.2014.08.008

Vitousek, P.M.; Naylor, R.L.; Crews, T.E.; David, M.B.; Drinkwater, L.E.; Holland, E.A.; Johnes, P.J.; Katzenberger, J.; Martinelli, L.A.; Matson, P.A. 2009. Nutrient imbalances in agricultural development. Science 324: 1519-1520. https://doi.org/ 10.1126/ science. 1170261

Wang, F.; Peng, S. 2017. Yield potential and nitrogen use efficiency of China's super rice. Journal of Integrative Agriculture 16: 1000-1008. https://doi.org/10.1016/ S2095-3119(16)61561-7

Wu, G.; Wilson, L.T.; McClung, A.M. 1998. Contribution of rice tillers to dry matter accumulation and yield. Agronomy Journal 90: 317-323. https://doi. org/10.2134/agronj1998.00021962009000030001x|

Wu, W.; Nie, L.; Liao, Y.; Shah F.; Cui, K.; Wang, Q.; Lian, Y.; Huang, J. 2013. Toward yield improvement of early-season rice: Other options under double ricecropping system in central China. European Journal of Agronomy 45: 75-86. https://doi.org/10.1016/j. eja.2012.10.009

Xia, Q.; Wang, L.; Xu, C.; Mei, J.; Li, Y. 2016. Effects of germination and high hydrostatic pressure processing on mineral elements, amino acids and antioxidants in vitro bioaccessibility, as well as starch digestibility in brown rice (Oryza sativa L.). Food Chemistry 214: 533542. https://doi.org/10.1016/j.foodchem.2016.07.114

Xie, K.; Guo, J.; Ward, K.; Luo, G.; Shen, Q.; Guo, S. 2020. The potential for improving rice yield and nitrogen use efficiency in smallholder farmers: a case study of Jiangsu, China. Agronomy 10: 419. https:// doi.org/10.3390/agronomy 10030419

Xu, L.; Zhan, X.; Yu, T.; Nie, L.; Huang, J.; Cui, K.; Wang, F.; Li, Y.S P. 2018. Yield performance of direct-seeded, double-season rice using varieties with short growth durations in central China. Field Crops Research 227: 49-55. https://doi.org/10.1016/j. fcr.2018.08.002

Yang, W.; Peng, S.; Laza, R.C.; Visperas, R.M.; Dionisiosese, M.L. 2007. Grain yield and yield attributes of new plant type and hybrid rice. Crop Science 47: 1393-1400. https://doi.org/10.2135/ cropsci2006.07.0457|

Yousaf, M.; Li, X.; Zhang, Z.; Ren, T.; Cong, R.; Ataulkarim, S.T.; Fahad, S.; Shah, A.N.; Lu, J. 2016. Nitrogen fertilizer management for enhancing crop productivity and nitrogen use efficiency in a riceoilseed rape rotation system in China. Frontiers in Plant Science 7: 1496-1496. https://doi.org/10.3389/ fpls.2016.01496 
Yuan, L.P. 2012. Conceiving of breeding further superhigh-yield hybrid rice. Hybrid Rice 6: 1-2. https:// doi.org/10.16267/j.cnki.1005-3956.2012.06.004 (in Chinese, with abstract in English).

Zhang, H.; Yu, C.; Hou, D.; Liu, H.; Zhang, H.; Tao, R.; Cai, H.; Gu, J.; Liu, L.; Zhang, Z. 2018. Changes in mineral elements and starch quality of grains during the improvement of japonica rice cultivars. Journal of the Science of Food \& Agriculture 98: 122-133. https://doi.org/10.1002/jsfa.8446

Zhang, T.; Yang, X. 2016. Mapping chinese rice suitability to climate change. The Journal of Agricultural Science 8: 33. http://dx.doi.org/10.5539/jas.v8n6p33

Zhang, Y.; Tang, Q.; Zou, Y.; Li, D.; Qin, J.; Yang, S.; Chen, L.; Xia, B.; Peng, S. 2009. Yield potential and radiation use efficiency of "super" hybrid rice grown under subtropical conditions. Field Crops Research 114: 91-98. https://doi.org/10.1016/j.fcr.2009.07.008
Zhong, X.; Peng, S.; Roland, J.B.; Huang, N.; Zheng, H. 2006. Some canopy indices influencing sheath blight development in hybrid rice. Chinese Journal of Rice Science 20: 535-542.

Zhou, J.; Gu, B.; Schlesinger, W.H.; Ju, X. 2016. Significant accumulation of nitrate in chinese semihumid croplands. Scientific reports 6: 25088. https:// doi.org/10.1038/srep25088

Zou, Y. 2011. Development of cultivation technology for double cropping rice along the Changjiang River valley. Scientia Agricultura Sinica 44: 254262 (in Chinese, with abstract in English). https:// doi:10.3864/i.issn.0578-1752.2011.02 